\title{
Second-price common-value auctions under multidimensional uncertainty ${ }^{\text {th }}$
}

\author{
Maria-Ángeles de Frutos ${ }^{\mathrm{a}}$, Lambros Pechlivanos ${ }^{\mathrm{b}, *}$ \\ a Department of Economics, Universidad Carlos III de Madrid, Calle Madrid 126, 28903 Getafe, Spain \\ ${ }^{\mathrm{b}}$ Department of International and European Economic Studies, Athens University of Economics and Business, \\ Patission 76, 10434 Athens, Greece
}

Received 20 February 2002

Available online 26 April 2005

\begin{abstract}
The literature has demonstrated that second-price common-value auctions are sensitive to the presence of asymmetries among bidders. In a two-bidder model, Bikhchandani [1988. Reputation in repeated second-price auctions. J. Econ. Theory 46, 97-119] has shown that if it is common knowledge that one bidder has a disadvantage compared to her opponent, that bidder (almost surely) never wins the auction. Employing a similar two-bidder model, this paper shows that this result does not carry over when one allows for two-sided uncertainty. In such case, in every equilibrium, the disadvantaged type bidder needs to win the auction with strictly positive probability. We then solve for the equilibria in two cases, one with two types and another with a continuum of types, and we show that they converge to the symmetric equilibria of the corresponding symmetric auctions. We thus reestablish a lost linkage in the analysis of common-value and almost-common-value auctions.
\end{abstract}

(c) 2005 Elsevier Inc. All rights reserved.

JEL classification: D44; D82; T96

Keywords: Common-value auctions; Asymmetric bidders; Spectrum auctions; Liquidity constraints

\footnotetext{
the first author's research was supported by the Spanish Ministerio de Educación (Grant DGICYTPB960118), and the second author's one by the European Commission under the TMR program (Grant ERBFMBICT961580).

* Corresponding author.

E-mail addresses: frutos@eco.uc3m.es (M.-Á. de Frutos), lpech@aueb.gr (L. Pechlivanos).
} 


\section{Introduction}

Auction design has been lately in vogue due to the recent wave of licensing for the third generation mobile telephony services (UMTS) throughout Europe. In those auctions, several countries established rules to counter potentially deleterious effects created by the presence of asymmetries among bidders. Asymmetries were considered to be prevalent because of the coexistence in the auctions of incumbent and entrant firms in the local telephony markets. ${ }^{1}$

Considerations about asymmetries among bidders come immediately into one's mind also when, for example, the object being auctioned off is a target firm in a takeover contest, a state-owned enterprise about to be privatized, or a bankrupt firm under liquidation. In all those cases, the market value of the assets is common to all bidders (i.e., they compete for common-value objects), but at the same time, each of them may have an additional private source of gains due to synergies.

Asymmetries among bidders can also be generated by the presence of liquidity constraints. Firms that operate within imperfect capital markets face different costs of raising the amount of cash needed for their bids. Differences in retained earnings, in values of assets appropriate for collateral, or, more generally, in access to external finance may easily cause asymmetries among bidders.

Auction theory, for reasons of technical convenience, had traditionally focused on symmetric environments. Nonetheless, attempts to extend the analysis to asymmetric environments have shown that most major insights derived in the symmetric case do not continue to hold. Perhaps, the most celebrated, violent reversal of fortune has been delivered in the analysis of single-object, second-price, common-value auctions. Bikhchandani (1988) has demonstrated that if in such an auction it is common knowledge that one of the bidders has a disadvantage, in a stochastic sense, compared to her opponent, this bidder (almost surely) never wins the auction. Klemperer (1998) delivered the same result in a deterministic asymmetric auction. As a consequence of these results, it is considered that second-price, common-value auctions may have considerably less desirable properties in asymmetric environments compared to symmetric ones; such as, not attracting potential bidders to the auction because they might feel intimidated by their opponents' prowess, or raising lower revenues in expectation to the seller. ${ }^{2}$ Actually, it is argued in Klemperer (2002) that these theoretical results have persuaded the designers of the spectrum auctions to establish rules that attempt to "level the field" for the entrants in the telephone markets, who were considered to be weaker.

This paper is an attempt to further our understanding on the properties of asymmetric second-price, common-value auctions. It departs from the existing literature in two ways. First, it introduces two-sided uncertainty, i.e., it analyzes the case in which both bidders' types are private information. This two-sided, stochastic departure from symmetry seems more natural than the extensions already considered in the literature. For

\footnotetext{
1 See Klemperer (2002).

2 Milgrom and Weber (1982) have shown that in symmetric common-value environments, second-price auctions, by reducing the winner's curse, generate larger expected revenues than first-price auctions do. Bikhchandani (1988) and Klemperer (1998) argue that this result does not carry over when asymmetries are introduced.
} 
it is highly unlikely that all additional bidders' characteristics, that cause asymmetries, are public information. One can easily appreciate this fact, when considering a model where asymmetries are due to liquidity constraints. Public financial statements, no matter how detailed or forthcoming they might be, never reveal all information available to management. But even in lopsided situations, assigning unequivocally the comparative advantage to a bidder is not a straightforward exercise. ${ }^{3}$ The second departure involves the explicit employment of discontinuous bidding strategies. ${ }^{4}$ Bidding strategies are not required to be continuous and strictly increasing.

Our more general framework considerably alters the outcomes derived in the literature. Even when the probabilities that one of the bidders is an advantaged type and the other a disadvantaged type are arbitrarily large, in equilibrium, the disadvantaged type bidder has to win the auction with strictly positive probability, even in the state of nature in which her opponent is actually an advantaged type. This result is delivered in generality in a two-type model (Proposition 1). Moreover, two applications are worked out explicitly; one in a twotype and another in a continuum-of-types model. In these applications it is actually shown that even if the stochastic comparative advantage a bidder enjoys is large she is not able to capitalize on it (Propositions 3 and 6). For example, in the two-type application, it is shown that when there is only $\epsilon$ probability for a bidder to be an advantaged type, while the other bidder is equally likely to be of an advantaged or a disadvantaged type, the first bidder wins the auction with probability equal to one fourth! Note that this probability of winning is computed when the state of nature is such that the first bidder is a disadvantaged type while her opponent an advantaged type. Clearly these results should alter one's perception about the ability of second-price auctions to attract bidders that could be intimidated by the presence of opponents, who may even have a substantial "stochastic comparative advantage."

It is also found that as we consider a sequence of auctions converging to the symmetric one, the equilibria we construct converge to the unique symmetric equilibrium of the symmetric auction (Propositions 4 and 7). These results restore the "common sense" intuition that a small advantage should not have an enormous impact on outcomes. We thus put into a different perspective the sharp discontinuity found in the literature as one moves from common-values to almost-common-values. Of course, it should be clear that continuity does not imply, in any sense, similarity in the equilibrium behavior of the bidders when confronted with different degrees of asymmetry.

Moreover, we show that the restriction to equilibria in continuous and strictly increasing strategies imposed in the literature is a necessary condition to derive the chasm between

\footnotetext{
3 For example, even in the electromagnetic spectrum auctions, where most observers assumed that incumbents enjoy a comparative advantage relative to entrants, one may surprisingly find difficulties in assigning "appropriate" tags to bidders. For example, Börgers and Dustmann (2003) report that TIW, an entrant that won a license in the UK $3 \mathrm{G}$ auction, was actually operating as a front company for Hutchison Whampoa, a corporation with presence in the UK mobile telephony market, which was not allowed to participate in the auction as an "entrant." Moreover, situations where small incumbents have been confronted with entrants who were established players in other large European telephone markets or domestic utilities with long nationwide presence in the country have been observed in some auctions.

4 Another recent exception to the rule of restricting attention to continuous bidding strategies is Fang and Parreiras (2002).
} 
results in symmetric and asymmetric auctions (Proposition 5). ${ }^{5}$ This way, we attempt to highlight the fact that this dichotomy was delivered due to specific characteristics in the extensions considered in the literature, and hence it can be misleading.

Of course, this analysis should not be interpreted as an innuendo for advocating a position against the importance of studying asymmetries among bidders. To the contrary, we first explain the importance of the way one goes about introducing asymmetries (i.e., adding a common knowledge or a stochastic comparative advantage). And then, and perhaps more importantly, we highlight the pedagogic importance the symmetric framework has as a limiting case when analyzing the properties of auctions in general settings.

In short, by considering an encompassing and more natural framework to introduce asymmetries among bidders, we reestablish a lost linkage in the analysis of pure-commonvalue and almost-common-value auctions. We believe that this is of importance not only for its aesthetic, theoretical appeal, but also because it brings back relevance to the intuitions gained by many theoretical results reached from the analysis of symmetric settings when considering real case studies, where, no doubt, asymmetries abound. For an econometrician, such result actually implies that it does make sense to test implications coming out of models on symmetric auctions; an otherwise meaningless exercise, according to the perceived in the literature fragility of the results in symmetric settings.

A related, but rather distinct, issue the paper is able to address is the issue of the effect the severity of financial constraints has on bidders' behavior. Until now the literature focused its analysis on budget constraints, which constitute a special form of the financial constraints. We show that, conditional on their types, bidders bid more aggressively when they operate in a more constrained environment. This result is due to the fact that, when a bidder faces opponents who are more likely to face more severe financial constraints, her winner's curse gets attenuated. ${ }^{6}$ Nonetheless, we show that one should not extrapolate from such result, and argue that a seller may be better off when her bidders operate in a more financially constraint environment. Although such counterintuitive result might be possible, we provide a wide class of distributions for which the seller is better off when her bidders are less financially constrained.

In recent years there is a renewed theoretical interest in the effects of the introduction of asymmetries in various auctions. As already mentioned, the first paper to study the effects of asymmetries in second-price common-value auctions was Bikhchandani (1988). Since then, it was Bulow and Klemperer who in a sequence of papers popularized the idea that the introduction of asymmetries greatly affects the outcome in a second-price auction in different contexts, and proposed ways to control for its effects. Klemperer (1998) analyzes numerous real life examples, such as the 1995 broadband spectrum auction in the US, in which he believes that the presence of asymmetries shaped the outcomes. Bulow et al. (1999) analyze the effects toeholds have on takeover battles. Finally, Bulow and Klemperer (2002) discuss how the ratio of the number of objects being auctioned off and of the number of bidders considered to be advantaged can be manipulated to mitigate the unwanted effects asymmetries create. In a private-value framework, Maskin and Riley (2000) per-

\footnotetext{
${ }^{5}$ In the two-type model, the constructed equilibrium involves discontinuous bidding strategies, which nonetheless converge to the standard continuous strategies of the symmetric auction.

6 A similar result was delivered by Fang and Parreiras (2002) in a model with budget constraints.
} 
form efficiency and revenue comparisons between first- and second-price auctions under different sorts of asymmetries in beliefs. Dasgupta and Maskin (2000) study the efficiency properties of various auctions, including one similar to ours (common values with multidimensional uncertainty). Finally, Che and Gale (1998) and Zheng (2001) study the effect of financial constraints on bidders' behavior in private-value auctions, while Fang and Parreiras (2002) consider the same issue, but in a common-value setting.

The paper is organized as follows. The next section sets up the model. Section 3 delivers the general results in the two-type case. Moreover, it explicitly solves for an asymmetric equilibrium (the only class of admissible candidate equilibria), and describes its convergence and expected revenues properties. Section 4 constructs a symmetric equilibrium in a continuum-of-types model and performs various comparative statics' exercises. Finally, Section 5 concludes.

\section{The model}

An auctioneer organizes an auction to sell an object. There are 2 risk-neutral bidders, $i \in\{I, I I\}$, competing to acquire it.

We assume that the value of the object is common to both bidders, and equals to $v \in$ $[\underline{v}, \bar{v}] \subset \mathbb{R}_{+}$. This value is unknown. Each bidder though receives a private signal $x_{i}$, such that $v=v\left(x_{I}, x_{I I}\right)=x_{I}+x_{I I}{ }^{7}$ The signals are assumed to be independently and identically distributed (i.i.d.) according to a cumulative distribution function $F$. The associated density function, $f$, is assumed to be absolutely continuous and strictly positive over its closed and bounded support $[0, \bar{x}] \equiv X \subset \mathbb{R}_{+} \cdot{ }^{8}$

Each bidder receives another private signal $\theta_{i} \in\left[\theta_{A}, \theta_{D}\right] \equiv \Theta \subset \mathbb{R}_{++}$, i.i.d. across bidders, distributed according to $G$, with absolutely continuous and strictly positive over its support density function, $g$. This signal contains private information regarding bidder $i$ 's marginal utility of income due to differential access to external sources of finance. ${ }^{9}$ Finally, there is no correlation between the two signals.

From now on, we will refer to $\theta_{i}$ as the type of bidder $i$, and to $x_{i}$ as the signal she has received (i.e., a bidder who has observed the pair $(x, \theta)$ will be referred to hereafter as a $\theta$-type bidder with signal $x$ ).

Bidder $i$ 's utility is $\widehat{U}_{i}=v-\theta_{i} p_{i}$. Nonetheless, instead of understanding the auction as one of pure common values in which bidders differ in their marginal utility of income, it is convenient to analyze it as an auction in which the bidders have identical marginal

\footnotetext{
7 The normalization that the value of the object is the sum of the signals is only made for notational and computational convenience. No results rely on it. An auction for such object is coined in the literature as the "wallet game."

8 The lower bound of the support is chosen to be zero to relieve notational burden. The extent to which this normalization affects the results will be explained as we proceed.

9 This is just one possible way to introduce asymmetries. Nonetheless, one can immediately see that the employed canonical model can be used to analyze the effects of asymmetries created by any other cause mentioned in Introduction.
} 
utilities of income, but they value the object differently. In this case, bidder $i$ 's utility can be rewritten as

$$
U_{i}=\frac{v}{\theta_{i}}-p_{i}
$$

Clearly, a bidder who has drawn a low $\theta$ has a comparative advantage compared to one who has observed a larger realization. Spanning through all possible realizations, the bidder with $\theta_{A}$ is the most advantaged-type bidder, and the one with $\theta_{D}$ is the most disadvantagedtype bidder.

The equilibrium notion we employ is stronger than the Bayesian-Nash equilibrium, as we impose the refinement the equilibrium to be in undominated strategies. In this two-bidder game, the equilibrium set in undominated strategies coincides with the set of trembling-hand-perfect equilibria (see Jackson, 1999). Essentially, this implies that a bid has to be at least as large as the minimum value of the object, given the signals the bidder received, i.e., $b_{i}\left(x_{i}, \theta_{i}\right) \geqslant x_{i} / \theta_{i} .{ }^{10}$ The set of bids satisfying the above condition with equality defines a plane on the $\left(x_{i}, \theta_{i}, b_{i}\right)$-space, which separates the set of all possible bids into two half-spaces. All bids belonging to the lower half-space, in which the above condition does not hold, allow for the possibility that the bidder may regret losing, regardless of the opponent's bidding strategy. The bidder can avoid this risk by placing a bid along the separating plane, without at the same time risking inducing a positive probability of regretting winning. In that sense, all bids in the lower half-space are dominated by bids lying on the separating plane, regardless of the opponent's bidding strategy, i.e., they guarantee the minmax payoff. Interpreted this way, the bids lying on the separating plane can be understood as the "individually rational" bids.

\section{Being disadvantaged is not fatal}

In this section, we consider that $\theta$ is distributed according to a two-point distribution $\Theta \equiv\left\{\theta_{A}, \theta_{D}\right\}$. A bidder who receives signal $\theta_{A}$ is called A-type, while if she receives $\theta_{D}$, D-type. ${ }^{11}$ Bidder $i$ is A-type with probability $\mu_{i}$, where $\mu_{i} \in(0,1)$, and D-type with the complementary probability. The bidder with the larger $\mu_{i}$ is the one who has the stochastic comparative advantage.

\subsection{General results}

The most striking result in the literature on asymmetric, second-price, common-value auctions is that a bidder who has a disadvantage compared to her opponent (almost surely) never wins the auction. In a model with one-sided uncertainty about one bidder's type, Bikhchandani (1988) shows that this result holds even if the probability that one bidder is more advantaged than the other is vanishingly small. In a deterministic framework, Bulow and Klemperer (2002) deliver the same result even when they allow the differences between types to become negligible.

\footnotetext{
10 This restriction is frequently made to rule out unreasonable equilibria, e.g., Bikhchandani (1988).

11 The notation $i$ - $j$ bidder, where $i=\{I, I I\}$ and $j=\{A, D\}$, denotes in this section a $j$-type bidder $i$.
} 
In this section, we show that this result is not robust to the introduction of two-sided uncertainty about the type of the two bidders. Specifically, we show that even if the probabilities that one of the bidders is A-type and the other D-type are arbitrarily large, it is possible that a D-type bidder wins the auction. It should be noted that she wins the auction with strictly positive probability even in the state of nature in which her opponent is A-type. This result holds regardless of whether the D-type bidder is the one considered to have the stochastic comparative advantage or not. In other words, we prove a more general version of the statement needed to show that the results shown in the literature do not carry over when one allows for two-sided uncertainty. Most notably, it holds even when $\mu_{I}$ is $\epsilon$ close to 1 and $\mu_{I I}$ is $\epsilon$ close to 0 , and the actual state of nature is the most probable one, i.e., bidder I is A-type and bidder II is D-type. The following proposition states formally the result. ${ }^{12}$

Proposition 1. For all $\left(\mu_{I}, \mu_{I I}\right)$, in every equilibrium in non-decreasing, pure strategies of this auction, there exists a D-type bidder who must win over an A-type bidder with strictly positive probability.

Proof. Assume not. In such case, in equilibrium, a D-type bidder wins with zero probability over an A-type bidder, and therefore an A-type bidder should assign zero probability to the event that she ties with a D-type bidder. Concentrate first on symmetric strategies. An A-type bidder must bid in equilibrium $b^{A}(x)=2 x / \theta_{A}$; i.e., the strategies derived in Milgrom (1981). In such equilibrium, for a D-type bidder to never win, she must bid $b^{D}(x)=0$. Consider a D-type bidder with signal $\bar{x}$. The bid coming out of her individually rational strategy is $\bar{x} / \theta_{D}$. By bidding it, she wins whenever $2 x / \theta_{A}<\bar{x} / \theta_{D}$. Hence, she wins against an A-type bidder with signal $x \in\left[0, \bar{x} \theta_{A} /\left(2 \theta_{D}\right)\right)$, while making positive profits conditional on winning. (The true value to her is $(\bar{x}+x) / \theta_{D}$, whereas she pays no more than $\bar{x} / \theta_{D}$.) Since this deviation is profitable, it contradicts equilibrium strategies.

Consider now asymmetric strategies. The proof is split into two cases: (a) We first show that there exists no equilibrium strategy in which one of the A-type bidders (say I-A) always wins over a positive mass of II-A bidders with signals $x \in S \subseteq X$. We have to analyze two situations: (i) II-A bidder uses an increasing strategy. As D-type bidders always lose, $b^{I I-A}(x) \geqslant \bar{x} / \theta_{D}, \forall x \in S$. Take $y=\bar{x} \theta_{A} / \theta_{D}$. Assume w.l.o.g. that $y \in S$. Consider the I-A bidder with signal $\epsilon$, where $\epsilon \rightarrow 0$. She is making losses when winning over a II-A bidder with signal $x \in[0, y)$. To see this, just notice that when winning the price is greater than the true value. Further, she does not make positive profits winning over a II-A bidder with signal larger than $y$, as such bidder bids at least her individual rational bid. Thus, she is better off by deviating down to $b^{I I-A}(0)$. (ii) II-A bidder's strategy has a flat interval, i.e., $\exists\left[x_{1}, x_{2}\right] \subset S$ such that $b^{I I-A}(x)=b, \forall x \in\left[x_{1}, x_{2}\right]$. For such strategy to be individually

\footnotetext{
12 The normalization that the lower bound in the support of $x$ is zero allows us to make the proposition statement unconditionally. Otherwise, it would have been necessary to assume that $(\bar{x}+\underline{x}) / \theta_{D}>2 \underline{x} / \theta_{A}$. That is, that the individually rational bid of the least advantaged type when receiving the highest possible signal is larger than the individually rational bid of the most advantaged type when receiving the lowest possible signal. This is nothing more than the weakest condition needed in an auction to ensure the presence of real competition among bidders. This condition is trivially satisfied when $\underline{x}=0$.
} 
rational, $b \geqslant x_{2} / \theta_{A}$. But then the I-A bidder with signal $\epsilon$ is better off by deviating down and not winning over any II-A bidder with signal $x \in\left[x_{1}, x_{2}\right]$.

(b) We now show that there exists no equilibrium strategy in which I-A and II-A bidders tie among themselves while D-type bidders always lose. Assume it does. Let II-A bidder use an increasing strategy. Consider again a I-A bidder with signal $\epsilon, \epsilon \rightarrow 0$. Assume that this bidder ties with a II-A bidder with signal $M$. Bertrand competition among them implies that $b^{I-A}(\epsilon)=(\epsilon+M) / \theta_{A} \geqslant \bar{x} / \theta_{D}$. As $\epsilon \rightarrow 0, M / \theta_{A} \geqslant \bar{x} / \theta_{D}$ which implies that $M \geqslant\left(\theta_{A} / \theta_{D}\right) \bar{x}>0$. Therefore, $M \nrightarrow 0$. Take a II-A bidder with signal $m<M$ who ties with a I-A bidder with signal $r$. In a tie, $(m+r) / \theta_{A} \geqslant \bar{x} / \theta_{D}$. As $m \rightarrow 0, r \geqslant\left(\theta_{A} / \theta_{D}\right) \bar{x}>\epsilon$; which contradicts increasing strategies. So if an equilibrium exists, the bidding strategies must have flat intervals. Employing similar arguments as in the previous case one can show that this is not possible.

The intuition behind the result is the following: An A-type bidder cannot bid very aggressively because she knows that with positive probability she may be playing against a bidder who may also be an A-type. If both are aggressive, they end up regretting when winning. Even if the probability of meeting an A-type is very small, the bidder's attention is concentrated on this event. This reluctance, on the other hand, makes the D-type bidders less cautious, and as a result the extreme situation analyzed in the literature is not obtained. D-type bidders need to win with strictly positive probability in all equilibria of the auction.

The next proposition further characterizes the set of admissible equilibria in this auction. Namely, it shows that there are no symmetric equilibria. ${ }^{13}$

\section{Proposition 2. There exist no symmetric equilibria in non-decreasing pure strategies.}

Proof. We first show that symmetric equilibrium bidding functions must be single-valued and continuous, i.e., there are no gaps. The proof is by contradiction. Assume w.l.o.g. that an A-type bidder's equilibrium strategy has a gap at $M$, i.e., $b^{A}(M)=b_{H}>b^{A}(M-\epsilon)=$ $b_{L}$. Two cases have to be considered: (a) Assume $\nexists x$ such that $b^{D}(x) \in\left(b_{L}, b_{H}\right)$. Conditional on winning, $\lim _{\epsilon \rightarrow 0} v(M-\epsilon, y)=v(M, y)$. Thus, either the bidder with signal $M$ makes strictly positive profits, and hence the bidder with signal $M-\epsilon$ has incentives to raise her bid; or she makes losses and hence has incentives to lower her bid. Note that if she makes zero profits, the bidder with signal $M-\epsilon$ makes profits and hence she has incentives to lower her bid. (b) Assume $\exists\left[x_{L}, x_{H}\right] \subset X$ such that $b^{D}(x) \in\left(b_{L}, b_{H}\right), \forall x \in\left[x_{L}, x_{H}\right]$. In a symmetric equilibrium, $b^{A}(x) \geqslant b^{D}(x), \forall x$. Just notice that for any price a D-type bidder with signal $x$ is willing to pay, an A-type bidder with the same signal makes strictly more profits. This implies that $x_{L}>M-\epsilon$. Given that the bidder with signal $M-\epsilon$ loses over these D-type bidders whereas the one with signal $M$ wins, she has incentives not to raise her bid only if: $E\left[b^{D}(y) \mid y \in\left[x_{L}, x_{H}\right]\right]=M+E\left[y \mid y \in\left[x_{L}, x_{H}\right]\right]>2 M$, where

\footnotetext{
13 Jackson (1999) provides an example with private and common value components in which a symmetric equilibrium does also fail to exist. His example is quite different from our setup as the common value component is either 0 or $v$ and the private component is either 0 or 1 with equal probability. Nevertheless, the intuition for the non-existence in his example is similar to ours. It is the impossibility to order bids in such a way that inferences from prices be monotonic; i.e., that larger bids generate larger expected values of the object in the event of ties.
} 
the inequality holds because $x_{L}>M-\epsilon$. Hence, in equilibrium $b^{A}(M)>2 M$. But then one of the A-type bidders, say bidder I, with signal $M$ can make more profits by lowering her bid, contradicting symmetric equilibrium. Hence, symmetric equilibria have to be in continuous strategies.

Assume now, by way of contradiction, that there exists an equilibrium in symmetric strategies. If this is the case, they have to be continuous. Further, an A-type bidder with signal $\bar{x}$ must win with probability 1 . Since by Proposition 1, a D-type bidder cannot always lose, and since strategies are shown to be continuous, there exists a signal $Z<\bar{x}$ such that $b^{A}(Z)=b^{D}(\bar{x})$. Now, the unique equilibrium strategy for an A-type bidder with signal greater than $Z$ is $b^{A}(x)=2 x / \theta_{A}, \forall x>Z$. The continuity of the strategies implies that $b^{A}(Z)=b^{D}(\bar{x})=2 Z / \theta_{A}$. Now, consider an A-type bidder with signal $Z-\epsilon$. If $b^{A}(Z-\epsilon)<2 Z / \theta_{A}$, then by deviating and bidding $2 Z / \theta_{A}$, she pays at most her bid, while the true value when tying with a bid $2 Z / \theta_{A}$ is $\left(1 / \theta_{A}\right)\left\{(Z-\epsilon)+\left(\mu_{i} Z+\left(1-\mu_{i}\right) \bar{x}\right)\right\}>$ $2 Z / \theta_{A}$. Since the deviation is profitable and strategies are assumed non-decreasing, it must be the case that $b^{A}(Z-\epsilon)=2 Z / \theta_{A}$. By the same argument, this must also be the bid of any A-type bidder with signal $y \in[z, Z]$, where $z$ is such that $z+\left\{\mu_{i}(E[x \mid x \in[z, Z]])+\right.$ $\left.\left(1-\mu_{i}\right) \bar{x}\right\}=2 Z$. Given that $z<Z$, one of the A-type bidders can make more profits by slightly increasing her bid. (The price she will pay is almost surely the same but she will increase the probability of winning.) Therefore, the deviation is profitable; a contradiction. This concludes the proof.

The logic behind the non-existence result can be deciphered by following the steps of the proof. The presence of asymmetries among bidders alters the nature of the winner's curse each bidder faces. For an A-type it gets milder due to the presence of D-type bidders: an A-type bidder may outbid opponents with signals higher than hers, who cannot bid higher because they are of D-type. (The opposite is true for a D-type bidder, her winner's curse gets stronger because of the presence of A-type bidders.) On the other hand, the probability that an A-type bidder places on the event that she ties with a D-type bidder decreases as her signal gets larger. As a result, the marginal A-type bidder, i.e., the one who ties with a D-type bidder with the highest signal, gets a less severe winner's curse than the A-type bidder with signal slightly above hers. Note that the former ties also with D-types, whereas the latter only ties with A-types. Because of this, the A-type marginal bidder is willing to place a strictly larger bid than an A-type bidder with slightly larger signal. Hence, an equilibrium in non-decreasing strategies cannot be obtained. ${ }^{14}$

Nonetheless, there exist asymmetric equilibria in such auction. The next subsection fully characterizes a pure-strategy, asymmetric equilibrium, and discusses its properties in an application with uniform distributions.

\footnotetext{
14 The presence of a critical bid, above which a D-type bidder never places her bid, is crucial for the nonexistence result. If there does not exist such bid, i.e., a bid in the neighborhood of which the distribution of types that place bids changes discretely, then equilibria in non-decreasing symmetric strategies may exist. In Section 4 , we provide such an equilibrium for a continuum-of-types model. There, as we consider higher bids, disadvantaged type bidders drop out of the auction, but this happens smoothly. As a result, no discrete jump in the "perceived" value of the object occurs, and hence the construction of non-decreasing symmetric equilibrium strategies is possible.
} 


\subsection{Asymmetric equilibria and their convergence properties}

\subsubsection{Characterization of an equilibrium}

Consider the following example: the $x_{i}$ 's are uniformly drawn from $[0,1], \theta_{A}=1$, and $\theta_{D}=2$.

The following proposition fully characterizes an asymmetric equilibrium in which a D-type bidder has a strictly positive probability of winning over an A-type bidder. ${ }^{15}$

Proposition 3. For all $\mu_{I}$, and for each $\mu_{I I}$ no larger than $1 / 2$, the following bidding strategies constitute an equilibrium:

$$
\begin{array}{ll}
\text { (I) } b^{I-A}(x)=\left\{\begin{array}{ll}
x+s & x \in[0, Q] \\
2 x & x \in(Q, 1]
\end{array},\right. & b^{I-D}(x)=\left\{\begin{array}{ll}
x & x \in[0, s] \\
x+s & x \in(s, 1]
\end{array},\right. \\
\text { (II) } b^{I I-A}(x)=\left\{\begin{array}{ll}
s & x \in[0, s] \\
2 M & x \in(s, M] \\
2 x & x \in(M, 1]
\end{array},\right. & b^{I I-D}(x)=\left\{\begin{array}{ll}
x & x \in[0, s] \\
s & x \in(s, 1]
\end{array},\right.
\end{array}
$$

where $s=\left(1-\mu_{I I}-\sqrt{\mu_{I I}-\mu_{I I}^{2}}\right) /\left(1-2 \mu_{I I}\right), M=(1+s) / 2$, and $Q=(3+s) / 4$.

Proof. We first show that there is no signal $x \in X$, such that I-A bidder, by deviating from the above strategies, can make more profits. If $x=0$, by following the purported equilibrium strategies, in the event of a tie, after updating her beliefs about the type of the opponent she tied with, she makes zero profits: Just notice that, by the definition of $s$, $\mu_{I I} s(s / 2-s)+\left(1-\mu_{I I}\right)(1-s)((1+s) / 2-s)=0$. Moreover, there is no other bid that gives her larger profits. If $x \in(0, Q)$, she makes positive profits by winning over a II-A bidder with signal in $[0, s]$ and over any II-D bidder. By deviating up to win over a II-A bidder with signal in $[s, M]$, she only succeeds in reducing her profits. Just notice that $x+(M+s) / 2-2 M<Q+(M+s) / 2-2 M=0$. When $x=Q$, she is indifferent between bidding $Q+s$ and $2 Q$. The only payoff-difference between these two strategies is that with the former she loses against a II-A bidder with signal in $[s, M]$, whereas with the latter she wins over her. Since, conditional on winning, expected profits are zero (just notice that $Q+(M+s) / 2-2 M=(3+s) / 4+(1+3 s) / 4-(1+s)=0)$, the two strategies are payoff-equivalent for the bidder. Clearly, if a I-A bidder with signal $Q$ is indifferent between winning or not over a II-A bidder with signal in $[s, M]$, a I-A bidder with signal larger than $Q$ does not regret winning. Hence, I-A bidder's strategy is best response against bidder II's strategies.

We now show that there is no signal $x \in X$, such that II-A bidder can profitably deviate from her purported equilibrium strategy. If $x \in[0, s)$, by following the strategy, she loses against any I-A bidder and a I-D bidder with signal in $[s, 1]$, but she does not regret losing. If she bids higher so as to win, she suffers a loss equal to $s-x>0$. When $x \in[s, M]$, she wins against a I-A bidder with signal in $[0, Q)$, and against any I-D bidder. She does not regret winning since, by doing so, she gains at least $x-s>0$. For any signal $x \in[s, Q$ ),

15 The presented equilibrium is not unique. Within the same spirit, more equilibria can be constructed. Nonetheless, they are more complex and they exist for a more restricted range of $\mu$ 's. 
she loses against a I-A with signal in $[Q, 1]$, but she does not regret losing. If she bids higher so as to win, she loses at least $x-Q>0$. Finally, when her signal is larger than $Q$ she follows the strategy $2 x$. By doing so, she wins over a I-A bidder with signal smaller than hers and makes profits upon outbidding them. By deviating up, to win over a I-A bidder with signal larger than hers, she makes loses. As she also makes profits by winning over a I-D bidder, there is no other strategy that gives her larger profits. Hence, no deviation is profitable.

Consider now D-type bidders. When they tie with their opponent, and according to the equilibrium strategies they assign zero probabilities to the event that they have tied with an A-type bidder, they play the unique symmetric equilibrium of an auction where: (a) it is common knowledge that both bidders have the same type, and (b) the signal space is truncated to contain only signals in which, by following the above specified strategies, they would have never tied with an A-type bidder. This ensures that the purported equilibrium strategies of D-type bidders with signal in $[0, s)$ are indeed equilibrium strategies. We now show that D-type bidders with larger signals do not want to deviate either. A I-D bidder with signal $s$ is indifferent between bidding $s$ and $2 s$. The only payoff-difference between these two strategies is that with the former she ties against a II-A bidder with signal in $[0, s]$ and against a II-D bidder with signal in $[s, 1]$, whereas with the latter she wins over both types. Since, conditional upon winning, expected payoffs are zero (just notice that, by the definition of $\left.s, \mu_{I I} s(3 s / 4-s)+\left(1-\mu_{I I}\right)(1-s)((3 s+1) / 4-s)=0\right)$, the two strategies are payoff-equivalent for this bidder. Since she is indifferent between tying or losing against those bidders, a I-D bidder with larger signal strictly prefers to win. Finally, notice that a I-D bidder with signal in $(s, 1]$ loses against a II-A bidder with signal larger than $s$, and she does not regret it.

Now a II-D bidder with signal in [ $s, 1]$ loses against a I-D bidder with signal larger than $s$ and against any I-A bidder. We now show that a II-D bidder with signal 1 does not want to deviate. She is willing to pay at most 1 . Given that a I-D bidder with signal $s$ bids $2 s>1$, she does not want to outbid her. Similarly, she does not regret losing against a I-A bidder with signal 0 , as the price she has to pay to win is $s$, while the true value conditional on winning is $1 / 2<s$. Clearly, if there is no profitable deviation for her, there cannot be any for a II-D bidder with a smaller signal.

We have proved that the preceding strategies are best responses against each other. Hence, they do constitute an equilibrium. Finally, notice that if $\mu_{I I}$ is no larger than $1 / 2$, then $s$ is no smaller than $1 / 2$. This means that, when bidding $s$, a II-D bidder with signal 1 bids more than her individual rational bid, which is $1 / 2$. Therefore, the strategies are individually rational.

It is worth highlighting an interesting property of the equilibrium: A D-type bidder has a strictly positive probability of winning the auction over an A-type bidder. Just notice that bidder I-D with signal $x \in[s, 1]$ beats bidder II-A with signal $x \in[0, s)$. Moreover, the equilibrium is independent of the actual values of $\mu_{I}$. So even if $\mu_{I}$ takes small values, the probability with which bidder I-D wins the auction over bidder II-A can be pretty large. This can be easily shown by checking the strategies in Fig. 1 .

For example, by setting $\mu_{I I}=1 / 2$ and $\mu_{I}=\epsilon$, one can find that the probability bidder I-D wins over bidder II-A is $1 / 4$. Hence, we do not speak about an $\epsilon$ probability event. 


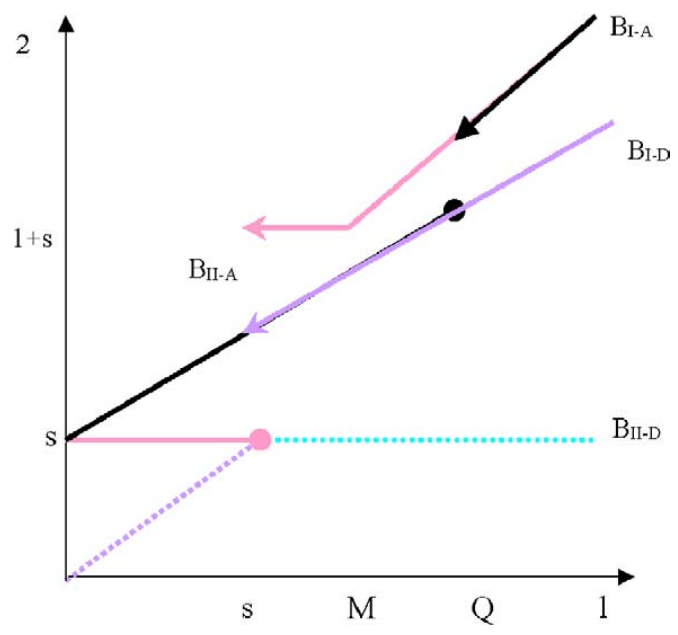

Fig. 1. Bidding strategies.

Even if the stochastic comparative advantage bidder II enjoys is large, this bidder cannot capitalize on this enhanced reputation and she ends up losing the auction with a large probability, even in the state of nature in which she is A-type playing against a D-type opponent.

The equilibria that arise in this model are qualitatively very different from a symmetric equilibrium. First of all, as it was derived in Proposition 2, they have to be in asymmetric strategies. Moreover, the bidding functions are neither strictly monotonic in the signals, and as it will become clear in the next subsection, nor in the stochastic comparative advantage.

Nevertheless, it should be stressed that the above constructed equilibrium also differs from the asymmetric equilibria of the symmetric auction. Note that these asymmetric equilibria of the symmetric auction have been heavily employed to derive the asymmetric equilibria found in models with deterministic comparative advantage (Bikhchandani, 1988 and Klemperer, 1998). At those asymmetric equilibria, a bidder is assigned an aggressive bidding strategy and her opponent a corresponding conservative strategy. The bidders do not alter their behavior throughout the signal space, i.e., the aggressive bidder remains aggressive regardless of receiving low or high signal. This is not the case here. For example, in the above described equilibrium, bidder I-A starts by being very aggressive when receiving a low signal, but then the degree of aggressiveness decreases, and for a range of intermediate signals, bidder II-A is the aggressive one.

Another novel characteristic of the equilibrium is that for a non-degenerate range of signals the equilibrium involves strategies that are symmetric. This is the case for the D-type bidders when receiving a low signal (less than $s$ ), and for the A-type bidders when receiving a high enough signal (more than $Q$ ). In that sense, it was deliberately attempted to construct the above described equilibrium as close as possible to a symmetric equilibrium. 


\subsubsection{Convergence properties}

It is of great importance to understand the behavior of the equilibrium as one considers the limit case in which the uncertainty about both or one of the bidders disappears. This can be studied by considering different scenarios. First, the two bidders may end up becoming similar. In this case the auction converges to a symmetric one with no uncertainty. Alternatively, the difference between the two bidders may become large. Finally, we will consider the case in which the auction converges to an asymmetric auction with one-sided uncertainty. Since the equilibrium strategies are independent of $\mu_{I}$, one can perform these exercises by just studying the strategies as $\mu_{I I}$ approaches zero.

Let $\left\langle\mu_{I I_{n}}\right\rangle$ be a decreasing sequence of real numbers with $\mu_{I I_{n}}>0$, and $\lim _{n \rightarrow \infty} \mu_{I I_{n}}=0$. Let $\Gamma_{\mu_{I}, \mu_{I I_{n}}}$ be the auction game in which bidder I is A-type with probability $\mu_{I}$ and bidder II with probability $\mu_{I I_{n}}$. For each $n$, and for every $\mu_{I}>0$, the set of strategies $\left\{b_{\mu_{I_{n}}}^{I-A}, b_{\mu_{I_{n}}}^{I I-A}, b_{\mu_{I_{n}}}^{I-D}, b_{\mu_{I_{n}}}^{I I-D}\right\}$ in Proposition 3 constitute an equilibrium of the game $\Gamma_{\mu_{I}, \mu_{I_{n}}}$, for $s_{n}=\left(1-\mu_{I I_{n}}-\sqrt{\mu_{I I_{n}}-\mu_{I I_{n}}^{2}}\right) /\left(1-2 \mu_{I I_{n}}\right), M_{n}=\left(1+s_{n}\right) / 2$ and $Q_{n}=\left(3+s_{n}\right) / 4$.

We first show that as the auction converges to a symmetric one in which both bidders are known to be D-type, the strategies in Proposition 3 converge to the symmetric equilibrium strategies "à la Milgrom," i.e., $b^{I-D}(x)=b^{I I-D}(x)=x$ for all $x \in[0,1]$.

Proposition 4. If $\Gamma_{\mu_{I}, \mu_{I I_{n}}} \rightarrow \Gamma_{0,0}$, then $b_{\mu_{I_{n}}}^{I-D}$ and $b_{\mu_{I_{n}}}^{I I-D}$ converge almost uniformly to Milgrom's symmetric equilibrium strategies.

Proof. As $\mu_{I I_{n}} \rightarrow 0, b_{\mu_{I_{n}}}^{I I-D}$ converges pointwise boundedly to $b^{I I-D}(x)=x$ for every $x \in[0,1]$, whereas $b_{\mu_{I_{n}}}^{I-D}$ converges a.e. to $b^{I-D}(x)=x$. Notice that it converges pointwise to the function $f(x)=x$ whenever $x \in[0,1]$ and to $f(x)=2$ whenever $x=1$. Hence, the result follows from Egoroff's theorem, as we have a sequence of measurable functions that converge a.e. on a bounded set to a continuous function. (See Royden, 1988, Chapter 3.)

This result should be contrasted to the one derived by Bikhchandani in his framework, where it is shown that the symmetric equilibrium strategy does not belong to the set of equilibrium strategies for a D-type bidder as one considers a sequence of auctions converging to a symmetric auction. ${ }^{16}$ Moreover, it puts under a different light the widely held belief that the introduction of the slightest asymmetry alters completely the analysis of a second-price, common-value auction. The symmetric auction is robust to small changes towards asymmetry in the following sense: When considering a sequence of auctions converging to the symmetric one, the asymmetric equilibrium we construct converges to the unique symmetric equilibrium of the symmetric auction. ${ }^{17}$

\footnotetext{
16 See Bikhchandani, 1988, Proposition 3, p. 111.

17 It is straightforward to show that the same continuity results also hold when we consider expected revenues. As $\mu_{I} \rightarrow \mu_{I I} \rightarrow 0$, expected revenues can be easily computed; they converge to $1 / 3$. Just notice that they are equal to the expected revenues coming out of the symmetric equilibrium of the symmetric auction in which both bidders are known to be D-type.
} 
We now turn to the case in which the auction converges to the "Bikhchandani

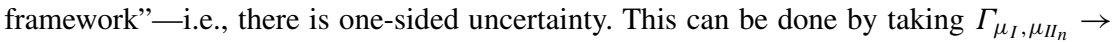
$\Gamma_{0, \mu_{I I}}$, i.e., $\mu_{I} \rightarrow 0$ and $\mu_{I I_{n}} \in(0,1 / 2)$. Now bidder I wins with strictly positive probability even in the event that her opponent is A-type. This divergence from the result reached by Bikhchandani is due to the fact that we have constructed an equilibrium with discontinuous and/or flat strategies while the analysis in Bikhchandani is confined to continuous and strictly increasing strategies. ${ }^{18}$

Proposition 5. Consider an asymmetric, common-value, second-price auction with onesided uncertainty. There does not exist an equilibrium in which a D-type bidder wins over an A-type opponent if and only if the equilibrium is in continuous and strictly increasing strategies.

Proof. Sufficiency is proved in Bikhchandani, 1988, Proposition 1, p. 108. Necessity is derived by the counterexample presented above.

In contrast, were we considering the opposite situation where $\Gamma_{\mu_{I}, \mu_{I I n}} \rightarrow \Gamma_{\mu_{I}, 0}$, i.e., $\mu_{I I_{n}} \rightarrow 0$, and $\mu_{I} \in(0,1)$, where the limiting equilibrium employs strictly increasing strategies, Bikhchandani's result would be valid. ${ }^{19}$

Finally, consider the case in which the auction converges to a deterministic asymmetric auction, as in Klemperer (1998). This is done by considering $\mu_{I} \rightarrow 1$ and $\mu_{I I} \rightarrow 0$. In this case the relevant strategies are those of A-type bidder I and D-type bidder II. It is clear that now the D-type bidder will never win. Hence, the equilibrium outcome of the auction converges almost uniformly to the one found in the literature. ${ }^{20}$

\section{Continuum-of-types case}

In this section we analyze the model when the distribution of $\theta$ is continuous. We first proceed to show that, unlike the two-type case, there exists now a symmetric equilibrium in continuous strategies. We then derive the convergence properties of the constructed equilibrium and we show that it converges to the symmetric equilibrium of the symmetric auction. We thus deliver, also in this case, the continuity result between pure-common-values and almost-common-values we established in the two-type case. Finally, we perform comparative statics' with respect to the distribution of the $\theta$ s. Such exercise is of interest because it allows us to see how the behavior of bidders gets affected by the severity of the financial constraints they face.

\footnotetext{
18 The next section, by considering the continuum-of-types case, constructs an equilibrium with the same qualitative properties as the one in Proposition 3, but in continuous and strictly increasing strategies. Hence, the main results delivered in this section do not hinge on the fact that we allow for discontinuities.

19 Note that as $\Gamma_{\mu_{I}, \mu_{I I_{n}}} \rightarrow \Gamma_{\mu_{I}, 0}$, all $b_{\mu_{I I_{n}}^{I-A}}, b_{\mu_{I I}}^{I-D}$, and $b_{\mu_{I I n} I I-D}$ converge almost uniformly to equilibrium strategies à la Bikhchandani. In particular, $b_{\mu_{I n}}^{I-A}(x) \rightarrow x+1, b_{\mu_{I I n}}^{I-D}(x) \rightarrow x$, and $b_{\mu_{I I n}}^{I I-D}(x) \rightarrow x$.

20 Note that as $\Gamma_{\mu_{I}, \mu_{I I n}} \rightarrow \Gamma_{1,0}, b_{\mu_{I I n}}^{I-A}$ and $b_{\mu_{I I}}^{I I-D}$ converge uniformly to asymmetric equilibrium strategies à la Milgrom. In particular, $b_{\mu_{I n}}^{I-A}(x) \rightarrow x+1$, and $b_{\mu_{I I n}}^{I I-D}(x) \rightarrow x$.
} 


\subsection{Characterization of the symmetric equilibrium}

We now characterize the equilibrium of the auction when $x$ is uniformly distributed on $[0, \bar{x}]$, and $\theta$ is distributed in the interval $\left[\theta_{A}, \theta_{D}\right]$ according to a c.d.f. $G$, with a continuous and differentiable density $g$. We further assume that $\theta_{D}-\theta_{A} \leqslant \theta_{A} .{ }^{21}$

We show that in this case, there exists a symmetric equilibrium in pure, continuous, and strictly monotone strategies, so that $b^{I}(x, \theta)=b^{I I}(x, \theta)=b(x, \theta), \forall x, \theta$.

Proposition 6. The following strategies constitute a symmetric equilibrium in pure, continuous, and strictly monotone strategies:

$$
b^{*}(x, \theta)=\frac{x+x^{E}(b)}{\theta},
$$

where

$$
x^{E}(b) \equiv E\left[x \mid(x, \theta) \in b^{*}(x, \theta)=b\right]=z_{1}(b) m(b)+\left(1-z_{1}(b)\right) M(b),
$$

with

$$
\begin{aligned}
& z_{1}(b)=w_{1}, \forall b \leqslant b^{*}\left(\bar{x}, \theta_{D}\right), \\
& m(b) \equiv\left[y \in X \mid b^{*}\left(y, \theta_{A}\right)=b\right], \quad \text { and } \\
& M(b) \equiv \begin{cases}{\left[y \in X \mid b^{*}\left(y, \theta_{D}\right)=b\right]} & \forall b \leqslant b^{*}\left(\bar{x}, \theta_{D}\right), \\
\bar{x} & \text { otherwise. }\end{cases}
\end{aligned}
$$

Proof. ${ }^{22}$ Any strategy that is part of a symmetric equilibrium has to be decreasing in $\theta$. Just notice, that for any price an $(x, \theta)$-bidder is willing to pay for the object, an $(x, \theta-\epsilon)$ bidder makes strictly more profits when outbidding the same set of opponents. Similarly, it is easy to see that they have to be increasing in $x$.

Further, in any symmetric equilibrium $b(0, \theta)=0, \forall \theta$. Suppose on the contrary that there exists a bid function $b(x, \theta)$ such that $(b, b)$ is an equilibrium and $b(0, \theta)=a>0$. When bidder I observes $x_{I}=a / 2$ and $\theta_{I}=\theta_{D}$, bidding 0 rather than the prescribed equilibrium bid (i.e., $a+\Delta a$ ) will only matter in the event of a winning bid. The probability of such event is positive. Now when bidding $a+\Delta a$ and winning, she obtains an expected profit which is bounded above by $\left(1 / \theta_{D}\right)(a / 2+a / 2)-a$. Notice that since strategies are decreasing in $\theta$, this bidder estimates the expected signal of her opponent as bounded above by $a / 2$. As the upper bound on her expected profit is negative, she is hence strictly better off by deviating down and bidding 0 , contradicting equilibrium strategies.

Let $\left(b^{\circ}, b^{\circ}\right)$ be a symmetric equilibrium. Let us define its common bidding range as bids below $\bar{B}$, where $\bar{B}=b^{\circ}\left(\bar{x}, \theta_{A}\right)$. Similarly, let $\widehat{B}=b^{\circ}\left(\bar{x}, \theta_{D}\right)$. Since $\left(b^{\circ}, b^{\circ}\right)$ is a symmetric

21 To characterize the equilibrium we assume that, conditional on a tie at $b$, the expected value of the object for a $\left(0, \theta_{A}\right)$-bidder is no larger than the expected value for an $\left(\bar{x}, \theta_{D}\right)$-bidder. A sufficient condition for this to hold is $\theta_{D}-\theta_{A} \leqslant \theta_{A}$. In Appendix A, we show how the result generalizes to other supports for which the above condition fails to hold. In particular, to facilitate the exposition we employ there an example where it is assumed that $x$ is uniformly distributed on $[0,1]$, whereas $\theta$ is uniformly distributed on [1,4].

22 We would like to thank an anonymous referee for proposing the use of line integrals to simplify the exposition of the proof, and to generalize an earlier statement of the proposition. 
equilibrium, for every $b \in[0, \widehat{B}]$, there exists a pair $m(b)$ and $M(b)$ in $[0, \bar{x}]$, such that $b=$ $b^{\circ}\left(m, \theta_{A}\right)$ and $b=b^{\circ}\left(M, \theta_{D}\right)$. Similarly, for every $b \in(\widehat{B}, \bar{B}]$ there exists $m(b) \in(0, \bar{x}]$ and $\tilde{\theta}(b) \in\left[\theta_{A}, \theta_{D}\right)$, such that $b=b^{\circ}\left(m, \theta_{A}\right)$ and $b=b^{\circ}(\bar{x}, \tilde{\theta})$.

We now show that a pair of $b^{*}(x, \theta)$ 's constitute a symmetric equilibrium. Suppose that bidder II adopts $b^{*}$ and consider the optimal response by bidder I. Every $(x, \theta)$-bidder I maximizes her expected profit by bidding up to the point where she is indifferent whether she is selected as a winner, when she is involved in a tie. Notice that such a bid maximizes her probability of winning, conditional on making non-negative profits when winning. Now, the true value of the object when bidding $b$ and facing a tie is $\left(x+x^{E}(b)\right) / \theta$, where $x^{E}(b)$ stands for the expected value of the bidder II's signal in the event of a tie, when bid$\operatorname{ding} b$. As $b^{*}(x, \theta)$ is equal to this expression, $\left(b^{*}, b^{*}\right)$ constitutes a symmetric equilibrium in continuous strategies.

To compute $x^{E}(b)$, we distinguish two cases:

(1) Bids below $\widehat{B}$. Thereafter, we will also refer to these bids as "low" bids. For any $b \leqslant \widehat{B}$, there is a set of points $(x, \theta)$ that belong to the iso-bid $b_{I I}^{*}(x, \theta)=b$, which we shall denote by $\Gamma=\{(x(t), \theta(t)): t \in[0,1]\}$. Consequently, $x^{E}(b)$ is the expectation of $X$ along a path, and is given by the following expression ${ }^{23}$ :

$$
x^{E}(b)=E[X \mid(X, \Theta) \in \Gamma]=\frac{\int_{0}^{1} x(t) f_{L}(x(t), \theta(t)) \sqrt{\left(x^{\prime}(t)\right)^{2}+\left(\theta^{\prime}(t)\right)^{2}} \mathrm{~d} t}{\int_{0}^{1} f_{L}(x(t), \theta(t)) \sqrt{\left(x^{\prime}(t)\right)^{2}+\left(\theta^{\prime}(t)\right)^{2}} \mathrm{~d} t},
$$

where $f_{L}(x, \theta)$ stands for the probability distribution of the types of a bidder in the isobid, i.e., the joint density of $X$ and $\Theta$. In particular, $f_{L}(x(t), \theta(t))=\frac{1}{x(1)-x(0)} g(\theta(t)){ }^{24}$ Consider the path $\left(m(b), \theta_{A}\right)$ and $\left(M(b), \theta_{D}\right)$ to be a simple arc. Since the set of all possible types $X \times \Theta$ is connected, any simple linear parameterization can be used to evaluate the path integral. In particular, we consider $x(t)=m(b)+(M(b)-m(b)) t$ and $\theta(t)=$ $\theta_{A}+\left(\theta_{D}-\theta_{A}\right) t$, for $0 \leqslant t \leqslant 1$. Consequently,

$$
x^{E}(b)=\frac{\int_{0}^{1}(m(b)+(M(b)-m(b)) t) \frac{g\left(\theta_{A}+\left(\theta_{D}-\theta_{A}\right) t\right)}{x(1)-x(0)} \sqrt{(M(b)-m(b))^{2}+\left(\theta_{D}-\theta_{A}\right)^{2}} \mathrm{~d} t}{\int_{0}^{1} \frac{g\left(\theta_{A}+\left(\theta_{D}-\theta_{A}\right) t\right)}{x(1)-x(0)} \sqrt{(M(b)-m(b))^{2}+\left(\theta_{D}-\theta_{A}\right)^{2}} \mathrm{~d} t} .
$$

After straightforward computations and integration by parts, one obtains that

23 An iso-bid in the $x \theta$-plane generates a path, i.e., a set of points $(x, \theta)$ for which

$$
x=\varphi(t), \quad \theta=\psi(t) \quad 0 \leqslant t \leqslant 1,
$$

where $\varphi(t), \psi(t) \in C$ for $0 \leqslant t \leqslant 1$. If $f: S \subseteq \mathbb{R}^{n} \rightarrow \mathbb{R}$ is a continuous scalar field and $C$ is a simple arc in $S$, then the integral of $f$ over $C$ is defined by

$$
\int_{C} f \mathrm{~d} s=\int_{0}^{1} f(\alpha(t))\left\|\alpha^{\prime}(t)\right\| \mathrm{d} t
$$

where $\alpha:[0,1] \rightarrow \mathbb{R}^{n}$ is any simple parameterization of $C$ (see Baxandall and Liebeck, 1986).

24 The results that follow can, alternatively, be derived from the fact that $b^{*}$ induces linear iso-bids, and hence omitting line integrals. 


$$
\begin{aligned}
x^{E}(b) & =\int_{0}^{1}(m(b)+(M(b)-m(b)) t) g\left(\theta_{A}+\left(\theta_{D}-\theta_{A}\right) t\right) \mathrm{d} t \\
& =M(b)-(M(b)-m(b)) \int_{0}^{1} G\left(\theta_{A}+\left(\theta_{D}-\theta_{A}\right) t\right) \mathrm{d} t \\
& =w_{1} m(b)+\left(1-w_{1}\right) M(b),
\end{aligned}
$$

where

$$
w_{1}=\int_{0}^{1} G\left(\theta_{A}+\left(\theta_{D}-\theta_{A}\right) t\right) \mathrm{d} t .
$$

Note that the weights on the end points of the path are solely determined by the distribution of $\theta$.

(2) Bids in $(\widehat{B}, \bar{B}]$. Thereafter, we will also refer to these bids as "high" bids.

For this set of bids we have,

$$
x^{E}(b)=\frac{\int_{0}^{1} x(t) f_{H}(x(t), \theta(t)) \sqrt{\left(x^{\prime}(t)\right)^{2}+\left(\theta^{\prime}(t)\right)^{2}} \mathrm{~d} t}{\int_{0}^{1} f_{H}(x(t), \theta(t)) \sqrt{\left(x^{\prime}(t)\right)^{2}+\left(\theta^{\prime}(t)\right)^{2}} \mathrm{~d} t},
$$

where now we have that $f_{H}(x, \theta)=\frac{1}{x(1)-x(0)} \frac{g(\theta(t))}{G(\theta(1))}$. Note that for these bids we must integrate over a path between $\left(m(b), \theta_{A}\right)$ and $(\bar{x}, \tilde{\theta}(b))$. By using an equivalent linear parameterization for the curve $x(t)=m(b)+(\bar{x}-m(b)) t$ and $\theta(t)=\theta_{A}+\left(\tilde{\theta}(b)-\theta_{A}\right) t$, for $0 \leqslant t \leqslant 1$, we get the following expression:

$$
\begin{aligned}
x^{E}(b) & =\frac{\int_{0}^{1}(m(b)+(\bar{x}-m(b)) t) \frac{g\left(\theta_{A}+\left(\tilde{\theta}(b)-\theta_{A}\right) t\right)}{G(\tilde{\theta}(b))(x(1)-x(0))} \sqrt{(\bar{x}-m(b))^{2}+\left(\tilde{\theta}(b)-\theta_{A}\right)^{2}} \mathrm{~d} t}{\int_{0}^{1} \frac{g\left(\theta_{A}+\left(\tilde{\theta}(b)-\theta_{A}\right) t\right)}{G(\tilde{\theta}(b))(x(1)-x(0))} \sqrt{(\bar{x}-m(b))^{2}+\left(\tilde{\theta}(b)-\theta_{A}\right)^{2}} \mathrm{~d} t} \\
& =\int_{0}^{1}(m(b)+(\bar{x}-m(b)) t) \frac{g\left(\theta_{A}+\left(\tilde{\theta}(b)-\theta_{A}\right) t\right)}{G(\tilde{\theta}(b))} \mathrm{d} t \\
& =\bar{x}-\left(\frac{\bar{x}-m(b)}{G(\tilde{\theta}(b))}\right) \int_{0}^{1} G\left(\theta_{A}+\left(\tilde{\theta}(b)-\theta_{A}\right) t\right) \mathrm{d} t \\
& =z_{1}(b) m(b)+\left(1-z_{1}(b)\right) \bar{x},
\end{aligned}
$$

where

$$
z_{1}(b)=\frac{1}{G(\tilde{\theta}(b))} \int_{0}^{1} G\left(\theta_{A}+\left(\tilde{\theta}(b)-\theta_{A}\right) t\right) \mathrm{d} t .
$$

One can check that as $b \rightarrow \bar{B}, m(b) \rightarrow m(\bar{B})=\bar{x}$, and hence $x^{E}(b) \rightarrow \bar{x}$. 
Finally, the existence of a continuous function $b^{*}$ that satisfies all the above equations can be easily shown by construction. Consider first an $(x, \theta)$-bidder such that $b^{*}(x, \theta) \in$ $\left[0, b^{*}\left(\bar{x}, \theta_{D}\right)\right]$. Let $b^{*}(x, \theta)=b$. The definition of $b^{*}$ implies that the following system of equations must hold:

$$
\begin{aligned}
& b-\frac{1}{\theta}\left(x+w_{1} m(b)+\left(1-w_{1}\right) M(b)\right)=0, \\
& b-\frac{1}{\theta_{A}}\left(m(b)+w_{1} m(b)+\left(1-w_{1}\right) M(b)\right)=0, \\
& b-\frac{1}{\theta_{D}}\left(M(b)+w_{1} m(b)+\left(1-w_{1}\right) M(b)\right)=0 .
\end{aligned}
$$

From the system, it is clear that both $m(b)$ and $M(b)$ are linear functions of $b$. Recall that $w_{1}$ only depends on $G$. Consequently, for each $(x, \theta)$ there exists a unique $b$ that solves the system. Further, the solution yields

$$
b^{*}(x, \theta)=\frac{2 x}{2 \theta-w_{1} \theta_{A}-\left(1-w_{1}\right) \theta_{D}},
$$

which is indeed increasing in $x$, and decreasing in $\theta$. Consider now an $(x, \theta)$-bidder, such that $b^{*}(x, \theta) \in\left(b^{*}\left(\bar{x}, \theta_{D}\right), b^{*}\left(\bar{x}, \theta_{A}\right)\right)$. Solving an equivalent system of equations, the solution yields

$$
b^{*}(x, \theta)=\frac{2 x}{2 \theta-z_{1}(b) \theta_{A}-\left(1-z_{1}(b)\right) \tilde{\theta}(b)} .
$$

Nonetheless, for these high bids, it is necessary to pin down the function $\tilde{\theta}(b)$. Let $b^{*}(x, \theta)=b$. By the definition of $b^{*}$ we know that the equilibrium bid of a $\left(m(b), \theta_{A}\right)$ type is equal to $b=\left(m(b)+x^{E}(b)\right) / \theta_{A}$, or equivalently, that $x^{E}(b)=\theta_{A} b-m(b)$. Since there exists a $(\bar{x}, \tilde{\theta}(b))$-type that submits the same bid, the following condition must also hold: $b=\left(\bar{x}+x^{E}(b)\right) / \tilde{\theta}(b)$. By combining the two, we can obtain an explicit expression for $\tilde{\theta}(b)$. In particular, we get that

$$
\tilde{\theta}(b)=\theta_{A}+\frac{\bar{x}-m(b)}{b} .
$$

Now in order for the equilibrium to exist, this $\tilde{\theta}(b)$ must be consistent with the proposed equilibrium: i.e., it must be decreasing in $b$, with $\tilde{\theta}\left(b^{*}\left(\bar{x}, \theta_{D}\right)\right)=\theta_{D}$ and $\tilde{\theta}\left(b^{*}\left(\bar{x}, \theta_{A}\right)\right)=$ $\theta_{A}$. It is easy to see that $\tilde{\theta}(b)$ is decreasing in $b$. Notice that

$$
\frac{\partial \tilde{\theta}(b)}{\partial b}=\frac{-m^{\prime}(b) b-(\bar{x}-m(b))}{b^{2}}<0, \quad \text { as } \bar{x} \geqslant m(b), \text { and } m^{\prime}(b) \geqslant 0, \forall b .
$$

Further,

$$
\begin{aligned}
& \tilde{\theta}\left(b^{*}\left(\bar{x}, \theta_{A}\right)\right)=\theta_{A}+\frac{\bar{x}-\bar{x}}{b^{*}\left(\bar{x}, \theta_{A}\right)}=\theta_{A}, \quad \text { and } \\
& \tilde{\theta}\left(b^{*}\left(\bar{x}, \theta_{D}\right)\right)=\theta_{A}+\frac{\bar{x}-m\left(b^{*}\left(\bar{x}, \theta_{D}\right)\right)}{b^{*}\left(\bar{x}, \theta_{D}\right)}=\theta_{A}+\frac{b^{*}\left(\bar{x}, \theta_{D}\right)\left(\theta_{D}-\theta_{A}\right)}{b^{*}\left(\bar{x}, \theta_{D}\right)}=\theta_{D} .
\end{aligned}
$$


To conclude the proof we have to show that the solutions of the two cases we derived above "glue" with each other, i.e., $b^{*}(x, \theta)$ is a continuous function in the entire range. To show this, consider the $\left(\bar{x}, \theta_{D}\right)$-type. The solution according to (1) is

$$
b^{*}\left(\bar{x}, \theta_{D}\right)=\frac{2 \bar{x}}{2 \theta_{D}-w_{1} \theta_{A}-\left(1-w_{1}\right) \theta_{D}}
$$

and according to (2) is

$$
b^{*}\left(\bar{x}, \theta_{D}\right)=\frac{2 \bar{x}}{2 \theta_{D}-z_{1}(b) \theta_{A}-\left(1-z_{1}(b)\right) \theta_{D}} .
$$

Notice that, as in this case $\tilde{\theta}(b)=\theta_{D}, z_{1}(b)$ becomes equal to $\left(1 / G\left(\theta_{D}\right)\right) \int_{0}^{1} G\left(\theta_{A}+\left(\theta_{D}-\right.\right.$ $\left.\left.\theta_{A}\right) t\right) \mathrm{d} t$, which reduces to $w_{1}$. This argument completes the proof.

Proposition 6 provides the characterization of a symmetric equilibrium in the continuum-of-types case. We have shown that equilibrium bid functions are piecewise linear functions that can be decomposed into "low" and "high" bids. It would be more informative to rewrite equilibrium "low" bids after undertaking the following change of variables, $y=\theta_{A}+\left(\theta_{D}-\theta_{A}\right) t$. Given that $w_{1} \equiv \int_{0}^{1} G\left(\theta_{A}+\left(\theta_{D}-\theta_{A}\right) t\right) \mathrm{d} t,(1)$ can be rewritten as

$$
b^{*}(x, \theta)=\frac{2 x}{2 \theta+\int_{\theta_{A}}^{\theta_{D}} G(y) \mathrm{d} y-\theta_{D}}=\frac{2 x}{2 \theta-E_{G}(\theta)},
$$

where the equality follows from integration by parts. Similarly, it is again more convenient to rewrite equilibrium "high" bids, by performing a similar as above transformation. Given that $z_{1}(b)=(1 / G(\tilde{\theta}(b))) \int_{0}^{1} G\left(\theta_{A}+\left(\tilde{\theta}(b)-\theta_{A}\right) t\right) \mathrm{d} t$, by undertaking the change of variables $y=\theta_{A}+\left(\tilde{\theta}(b)-\theta_{A}\right) t$, Eq. (2) becomes

$$
b^{*}(x, \theta)=\frac{2 x}{2 \theta+\frac{1}{G(\tilde{\theta}(b))} \int_{\theta_{A}}^{\tilde{\theta}(b)} G(y) \mathrm{d} y-\tilde{\theta}(b)}=\frac{2 x}{2 \theta-E_{G}\left[\theta \mid \theta \leqslant \tilde{\theta}^{G}(b)\right]},
$$

where again the equality follows from integration by parts. It is now straightforward to see that the equilibrium bid functions are given by

$$
b^{*}(x, \theta)= \begin{cases}\frac{2 x}{2 \theta-E_{G}(\theta)} & \text { if } x \in\left[0, \frac{\bar{x}\left(2 \theta-E_{G}(\theta)\right)}{2 \theta_{D}-E_{G}(\theta)}\right], \\ \frac{2 x}{2 \theta-E_{G}\left[\theta \mid \theta \leqslant \tilde{\theta}^{G}(b)\right]} & \text { if } x \in\left[\frac{\bar{x}\left(2 \theta-E_{G}(\theta)\right)}{2 \theta_{D}-E_{G}(\theta)}, \bar{x}\right] .\end{cases}
$$

To better understand the properties of the equilibrium bid functions, we present an example in which both $\theta$ and $x$ are uniformly distributed, and moreover we set $\bar{x}=1, \theta_{A}=1$, and $\theta_{D} \leqslant 2$. It is now straightforward to calculate the equilibrium bid functions:

$$
b^{*}(x, \theta)= \begin{cases}\frac{4 x}{4 \theta-\theta_{D}-1} & \text { if } x \in\left[0, \frac{4 \theta-\theta_{D}-1}{3 \theta_{D}-1}\right], \\ \frac{3 x+1}{3 \theta-1} & \text { if } x \in\left[\frac{4 \theta-\theta_{D}-1}{3 \theta_{D}-1}, 1\right] .\end{cases}
$$

Note that the bid functions are piecewise linear in $x$. They have a steeper slope in the first segment, and moreover this slope is decreasing in $\theta$. The fact that the slope is decreasing in $\theta$ shows that the more efficient a bidder is (i.e., the smaller her $\theta$ is), the more aggressively she bids. 
An alternative approach to further understand and interpret the equilibrium bids is to compute the density mass of the equilibrium iso-bids. This allows us to see how likely it is that a particular bid is submitted. Denote as $\beta^{G}(b)$ the density mass of the iso-bid corresponding to $b_{G} \cdot{ }^{25}$

Consider first low equilibrium bids, i.e., $b_{G}(x, \theta)=2 x /\left(2 \theta-E_{G}(\theta)\right)$. By reparameterizing and using that $(x(\theta), \theta)=\left(b\left(2 \theta-E_{G}(\theta)\right) / 2, \theta\right)$, we get that

$$
\beta^{G}(b)=\int_{\theta_{A}}^{\theta_{D}} \frac{g(\theta)}{\bar{x}} \sqrt{1+b^{2}} \mathrm{~d} \theta=\frac{\sqrt{1+b^{2}}}{\bar{x}} .
$$

Notice that for low bids, $\beta^{G}(b)$ does not depend upon $G$. This is the case because low bids may be submitted from bidders whose $\theta$ can take any value in $\left[\theta_{A}, \theta_{D}\right]$, and moreover, because the mass of signals that can produce the bid, measured by $M(b)-m(b)$, is independent of $G$. Consequently, the mass of types along an iso-bid that represents a low bid is not affected by $G$. As a result throughout the whole range of low equilibrium bids the winner's curse remains unchanged. ${ }^{26}$

For high bids, in contrast, the distribution of $\theta$ affects the density of types on a given equilibrium bid, which is now given by

$$
\beta^{G}(b)=\int_{\theta_{A}}^{\tilde{\theta}^{G}(b)} \frac{g(\theta)}{\bar{x}} \sqrt{1+b^{2}} \mathrm{~d} \theta=\frac{\sqrt{1+b^{2}}}{\bar{x}} G\left(\tilde{\theta}^{G}(b)\right) .
$$

Notice that now it is not the case that all types of bidders may submit these bids. Bidders with $\theta>\tilde{\theta}^{G}$ are not present. Hence, the specific form of $G$ affects the mass of types that may be submitting a particular bid. Moreover, the larger is the bid we consider, the smaller is the set of bidders that can possibly submit such bid, and the larger is the mass of rather advantaged bidders in this distribution. As a result the winner's curse gets attenuated.

\subsection{Convergence to symmetric auction}

It is of particular interest to understand the behavior of the equilibrium as one considers the limit case in which the asymmetries among bidders disappear and the auction converges to a symmetric one. This is accomplished by performing comparative statics, as we let the distribution of $\theta, G$, converge to a Dirac delta distribution. By fixing the "spike" or the mass point at $\theta=\theta_{C}$, we can study the limiting behavior of the equilibrium bids as $\left\{G_{n}(\theta)\right\} \rightarrow \delta_{\theta_{C}}$, where $\theta_{A} \leqslant \theta_{C} \leqslant \theta_{B}$. In the limit, the equilibrium strategies converge to the symmetric equilibrium strategies of the symmetric auction, i.e., $\lim _{\theta \rightarrow \theta_{C}} b^{*}(x, \theta)=$ $b^{*}\left(x, \theta_{C}\right)=2 x / \theta_{C}, \forall x$.

\footnotetext{
25 We now explicitly denote that bids are functions of $G$, the distribution of $\theta$.

26 To avoid any misinterpretation, we would like to stress that this result does not imply that the equilibrium bids are independent of the distribution of $\theta$. As it will be shown in Section 4.3, the distribution of low equilibrium bids is affected by $G$.
} 
Proposition 7. For all $\theta_{C} \in\left[\theta_{A}, \theta_{D}\right]$, as $\left\{G_{n}(\theta)\right\} \rightarrow \delta_{\theta_{C}}$ in distribution, the symmetric equilibrium strategies of the asymmetric auction converge pointwise to the symmetric equilibrium strategies of the symmetric auction.

Proof. The analysis is split in two cases: (a) Low bids, i.e., bids below $\widehat{B}$, in which equilibrium bids are determined by Eq. (3), and (b) high bids, i.e., bids in $(\widehat{B}, B]$, in which equilibrium bids are determined by Eq. (4). Consider the first case: According to (3), $b^{*}(x, \theta)=2 x /\left(2 \theta-\int_{\theta_{A}}^{\theta_{D}} y g(y) \mathrm{d} y\right)$. Using the fact that the delta function obeys the shifting property: $\int_{a}^{b} f(y) \delta\left(y-\theta_{C}\right) \mathrm{d} y=f\left(\theta_{C}\right)$ (see Bracewell, 1999, pp. 74-75), we have that $\int_{\theta_{A}}^{\theta_{D}} y g(y) \mathrm{d} y \rightarrow \theta_{C}$, which implies $b^{*}\left(x, \theta_{C}\right) \rightarrow 2 x / \theta_{C}$. Consider now the second case. According to (4),

$$
b^{*}(x, \theta)=\frac{2 x}{2 \theta-\left(\int_{\theta_{A}}^{\tilde{\theta}(b)} y g(y) \mathrm{d} y / G(\tilde{\theta}(b))\right)} .
$$

Using again the shifting property of the Dirac function we have that $\int_{\theta_{A}}^{\tilde{\theta}(b)} y g(y) \mathrm{d} y \rightarrow \theta_{C}$ and $G(\tilde{\theta}(b)) \rightarrow 1$, which implies $b^{*}\left(x, \theta_{C}\right) \rightarrow 2 x / \theta_{C}$. Nonetheless, note that now these results rely on the fact that $\tilde{\theta}(b)$ converges to $\theta_{C}$. To prove this claim, we derive the limiting behavior of $z_{1}(b)$. By its definition, as $\left\{G_{n}(\theta)\right\} \rightarrow \delta_{\theta_{C}}$,

$$
z_{1}(b) \equiv \frac{\int_{0}^{1} G\left(\theta_{A}+\left(\tilde{\theta}(b)-\theta_{A}\right) t\right) \mathrm{d} t}{G(\tilde{\theta}(b))} \rightarrow \frac{\tilde{\theta}(b)-\theta_{C}}{\tilde{\theta}(b)-\theta_{A}} .
$$

On the other hand, by solving Eq. (2) for $z_{1}(b)$, when $\theta=\tilde{\theta}$ and $x=\bar{x}$, we get that $z_{1}(b)=$ $(2 \bar{x} / b-\tilde{\theta}(b)) /\left(\tilde{\theta}(b)-\theta_{A}\right)$. Since from the first part of the proof we know that $2 \bar{x} / b \rightarrow \theta_{C}$, the right-hand side of the above equation converges to $\left(\theta_{C}-\tilde{\theta}(b)\right) /\left(\tilde{\theta}(b)-\theta_{A}\right)$. Hence, a necessary condition for the above equation to hold in the limit is that $\tilde{\theta}(b) \rightarrow \theta_{C}$. This argument completes the proof.

Comparing Propositions 4 and 7, one can check that the results delivered in the twotype case were not due to the fact that we do not restrict attention to continuous strategies. This can be easily understood by Proposition 7: Although the equilibrium strategies are continuous and strictly increasing, the properties of the equilibrium have nothing to do with the results found in the literature. To the contrary, the equilibrium is qualitatively similar to the one reached in the two-type case. In both cases, as we consider a sequence of auctions converging to the symmetric one the equilibrium converges to the symmetric equilibrium of the symmetric auction. Remember that according to Proposition 3 in Bikhchandani (1988), the symmetric equilibrium does not belong to the set of equilibria, as one considers a sequence of auctions converging to the symmetric auction.

\subsection{Severity of financial constraints}

Another comparative statics' exercise that is interesting to perform is to check how bidders' behavior is affected when considering changes in the distribution of $\theta \mathrm{s}(G)$, and which are the repercussions on seller's expected revenues. To understand why this exercise 
is of interest one has to remember that $\theta$ can be understood as the marginal cost of raising money to finance a bid. In that sense, distributions that place more mass on higher values of $\theta$ represent more financially constrained bidders. Hence, when considering two possible distributions of $\theta, G$ and $H$, the assumption that $G$ first-order stochastically dominates $H$ is equivalent to saying that bidders under $G$ are more financially constrained than under $H$.

\subsubsection{Equilibrium bid functions}

We first analyze how the equilibrium bid functions are affected by the severity of financial constraints. The result is that, conditional on her type, a bidder bids more aggressively when all bidders are more financially constrained. The intuition is rather clear, when her opponents are more financially constrained, her winner's curse gets attenuated and she bids more aggressively. This comes at odds with the result derived by Che and Gale (1998), where a bidder of specific type bids the same. The difference between this paper and Che and Gale (1998) is twofold: First, they consider budget constraints, and second, they consider a private values framework. ${ }^{27}$ Fang and Parreiras (2002) showed that it was the private values assumption that allowed Che and Gale to reach this conclusion. By considering a framework with budget constraints and affiliated values, they derived the same result as we do.

Nonetheless, it has to be stressed that the use of budget constraints, instead of the more general financial constraints we employ, also affects the result. When considering budget constraints, more financially constrained bidders are those with smaller budgets. Nonetheless, for low bids, i.e., bids such that none of the bidders' budget constraints are binding, all bidders are symmetric, in the sense that they all have the same cost of financing a bid. Here in contrast, bidders' behavior is always affected (no matter how small the bid a bidder contemplates in placing) by the severity of her financial constraint. This is the reason why we get our result, even when we restrict attention to the low bids, while Fang and Parreiras (2002) get for low bids the result derived in Che and Gale (1998). The following proposition delivers formally the result. ${ }^{28}$

Proposition 8. Let $G$ and $H$ belong to the family of log-concave distributions. If $G$ strictly second-order stochastically dominates $(S S D) H$, then $b_{H}^{*}(x, \theta)<b_{G}^{*}(x, \theta)$, for all $(x, \theta)$ such that $x \in(0, \bar{x})$ and all $\theta$.

Proof. For any given distribution of $\theta$ we have shown that the support of equilibrium bids is given by $[0, \bar{B}]$. Further, this set comprises low and high bids, in such a way that $[0, \bar{B}]=\left[0, \widehat{B}_{F}\right] \cup\left[\widehat{B}_{F}, \bar{B}\right]$, where $\widehat{B}_{F}=b_{F}^{*}\left(\bar{x}, \theta_{D}\right) .{ }^{29}$

\footnotetext{
27 Budget constraints can be understood as special cases of our framework, in which the marginal cost of financing a bid is one until the bidder reaches her budget constraint and then becomes infinite.

28 Although in the analysis we present the comparative statics in terms of first-order stochastic dominance, as this is the most transparent way to provide an intuition for the result, the proposition does not require such a strong condition and it is proved for second-order stochastic dominance. Note that first-order stochastic dominance implies second-order stochastic dominance.

29 Hereafter, we denote the bid that separates low and high bids as $\widehat{B}_{F}$, where $F$ stands for a generic distribution of $\theta$, in order to underline the fact that it depends on the employed distribution. The same convention is employed for other variables when necessary.
} 
Consider first low bids. The distribution of $\theta$ affects bidding behavior via its impact on $w_{1}^{F}$. Remember that $w_{1}^{F} \equiv \int_{0}^{1} F\left(\theta_{A}+\left(\theta_{D}-\theta_{A}\right) t\right) \mathrm{d} t$. By the definition of strict SSD, $G$ SSD $H$ if and only if $\int_{\theta_{A}}^{\theta} H(t) \mathrm{d} t>\int_{\theta_{A}}^{\theta} G(t) \mathrm{d} t$, for all $\theta>\theta_{A}$. After a straightforward change of variables, we get that this implies that

$$
w_{1}^{H}=\frac{1}{\left(\theta_{D}-\theta_{A}\right)} \int_{\theta_{A}}^{\theta_{D}} H(y) \mathrm{d} y>\frac{1}{\left(\theta_{D}-\theta_{A}\right)} \int_{\theta_{A}}^{\theta_{D}} G(y) \mathrm{d} y=w_{1}^{G} .
$$

Since $b_{F}^{*}$ is a decreasing function in $w_{1}^{F}$, two results follow: First, $\widehat{B}_{H}<\widehat{B}_{G}$, and second, $b_{H}^{*}(x, \theta)<b_{G}^{*}(x, \theta)$, for all $b^{*} \in\left[0, \widehat{B}_{H}\right]$.

Consider now bids in $\left[\widehat{B}_{G}, \bar{B}\right]$, i.e. bids in the set of high bids for both distributions. By Eq. (4), we have

$$
b_{F}^{*}(x, \theta)=\frac{2 x}{2 \theta-E_{F}\left[\theta \mid \theta \leqslant \tilde{\theta}^{F}(b)\right]} .
$$

Thus, in this case, the distribution of $\theta$ affects bidding behavior through its impact on $E_{F}\left[\theta \mid \theta \leqslant \tilde{\theta}^{F}(b)\right]$. Specifically, $b_{G}^{*}(x, \theta)>b_{H}^{*}(x, \theta)$ if and only if $E_{G}\left[\theta \mid \theta \leqslant \tilde{\theta}^{G}(b)\right]>$ $E_{H}\left[\theta \mid \theta \leqslant \tilde{\theta}^{H}(b)\right]$. Now, for a given, arbitrary $\tilde{\theta}(b)$, Ogryczak and Ruszczynski (2002) show $^{30}$ that if $G \operatorname{SSD} H$, then $E_{G}[\theta \mid \theta \leqslant \tilde{\theta}(b)] \geqslant E_{H}[\theta \mid \theta \leqslant \tilde{\theta}(b)]$. Moreover, since $E_{F}[\theta \mid \theta \leqslant \tilde{\theta}(b)]$ is strictly increasing in $\tilde{\theta}(b)$, if $\tilde{\theta}^{G}(b)>\tilde{\theta}^{H}(b)$, then $E_{G}[\theta \mid \theta \leqslant$ $\left.\tilde{\theta}^{G}(b)\right]>E_{G}\left[\theta \mid \theta \leqslant \tilde{\theta}^{H}(b)\right] \geqslant E_{H}\left[\theta \mid \theta \leqslant \tilde{\theta}^{H}(b)\right]$. This will allow us to conclude that $b_{G}^{*}(x, \theta)>b_{H}^{*}(x, \theta)$. In what follows we show that this is indeed the case, as indeed $\tilde{\theta}^{G}(b)>\tilde{\theta}^{H}(b)$. To show this, consider the implicit equation that determines $\tilde{\theta}^{F}(b)$ :

$$
\frac{2 \bar{x} / b-\tilde{\theta}^{F}(b)}{\tilde{\theta}^{F}(b)-\theta_{A}}=\frac{\int_{0}^{1} F\left(\theta_{A}+\left(\tilde{\theta}^{F}(b)-\theta_{A}\right) t\right) \mathrm{d} t}{F\left(\tilde{\theta}^{F}(b)\right)},
$$

or equivalently,

$$
\frac{2 \bar{x}}{b}-\tilde{\theta}^{F}(b)=\tilde{\theta}^{F}(b)-E_{F}\left[\theta \mid \theta \leqslant \tilde{\theta}^{F}(b)\right] .
$$

Thus, for a given $b$, and for any $G$ and $H$, if $G \operatorname{SSD} H$, then

$$
\begin{aligned}
& \frac{2 \bar{x}}{b}=2 \tilde{\theta}^{G}(b)-E_{G}\left[\theta \mid \theta \leqslant \tilde{\theta}^{G}(b)\right] \leqslant 2 \tilde{\theta}^{G}(b)-E_{H}\left[\theta \mid \theta \leqslant \tilde{\theta}^{G}(b)\right], \\
& \frac{2 \bar{x}}{b}=2 \tilde{\theta}^{H}(b)-E_{H}\left[\theta \mid \theta \leqslant \tilde{\theta}^{H}(b)\right] .
\end{aligned}
$$

Since $2 \tilde{\theta}^{H}(b)-E_{H}\left[\theta \mid \theta \leqslant \tilde{\theta}^{H}(b)\right] \leqslant 2 \tilde{\theta}^{G}(b)-E_{H}\left[\theta \mid \theta \leqslant \tilde{\theta}^{G}(b)\right]$, it follows from the log-concavity of both $G$ and $H$ that $\tilde{\theta}^{H}(b) \leqslant \tilde{\theta}^{G}(b){ }^{31}$

\footnotetext{
30 See Theorem 3.2 in Ogryczak and Ruszczynski (2002).

31 The function $\delta(x)=x-E_{F}[y \mid y \leqslant x]$ is monotonically increasing in $x$ if and only if $J(x)=\int_{l}^{x} F(y) \mathrm{d} y$ is $\log$-concave. Now, the log-concavity of $J$ is implied by the log-concavity of $f(y)$ or of $F(y)$ (see Theorem 3 in Bagnoli and Bergstrom, 1989).
} 
Finally, for bids in $\left[\widehat{B}_{H}, \widehat{B}_{G}\right]$ the result follows trivially from the continuity and strict monotonicity of the equilibrium bids, which given the above results, do not allow a situation in which $b_{H}^{*}>b_{G}^{*}$ to arise.

\subsubsection{Expected revenues}

Until now we have shown that a bidder of a given type $(x, \theta)$ bids more aggressively when bidders are more likely to be disadvantaged, i.e., she bids more under $G$ than under $H$. This is a rather counterintuitive result given that it means that bidders in more financially constrained environments bid more aggressively. It should be stressed though that this result does not carry over in a straightforward way when we consider the effects financial constraints have on seller's expected revenues. I.e., one cannot extend the previous argument and say that expected revenues are larger under $G$ than under $H$, as the distribution of types depends on which is the distribution of $\theta$. In fact, in our setup, under $H$ any given type bids less but types that bid high (i.e., those with low $\theta$ ) become more frequent. Notice that the probability of having advantaged bidders equals to $H(\theta)$, which is larger than $G(\theta)$. As these two effects go in opposite directions, we cannot unconditionally conclude that seller's expected revenues are lower under $H$, which would have been tantamount to saying that the seller benefits when her bidders are more financially constrained.

To compute the expected revenues we need the distribution of the equilibrium bids. Note that the probability that a bid smaller or equal than $b$ is realized can be easily calculated by integrating over all iso-bid curves up to the one representing $b$. For low bids, up to $\widehat{B}^{F}$, we have:

$$
D^{F}(b)=\int_{\theta_{A}}^{\theta_{D}} \frac{b}{2 \bar{x}}\left(2 \theta-E_{F}(\theta)\right) f(\theta) \mathrm{d} \theta=\frac{b E_{F}(\theta)}{2 \bar{x}} .
$$

Now, by using the fact that if $G \operatorname{SSD} H$ then $E_{G}(\theta) \geqslant E_{H}(\theta)$, we obtain that $D^{G}(b) \geqslant$ $D^{H}(b)$.

For high bids in $\left[\widehat{B}^{F}, \bar{B}\right]$ we get that

$$
\begin{aligned}
D^{F}(b) & =1-F\left(\tilde{\theta}^{F}(b)\right)+\int_{\theta_{A}}^{\tilde{\theta}^{F}(b)} \frac{b}{2 \bar{x}}\left(2 \theta-E_{F}\left[\theta \mid \theta \leqslant \tilde{\theta}^{F}(b)\right]\right) f(\theta) \mathrm{d} \theta, \\
& =1-F\left(\tilde{\theta}^{F}(b)\right)+\frac{b}{2 \bar{x}} \int_{\theta_{A}}^{\tilde{\theta}^{F}(b)} \theta f(\theta) \mathrm{d} \theta=1-\frac{b}{\bar{x}} \int_{\theta_{A}}^{\tilde{\theta}^{F}(b)} F(\theta) \mathrm{d} \theta,
\end{aligned}
$$

where last equality follows from the definition of high bids, which implies that $(b / 2 \bar{x})$ $\left(\tilde{\theta}^{F}(b) F\left(\tilde{\theta}^{F}(b)\right)+\int_{\theta_{A}}^{\tilde{\theta}^{F}(b)} F(\theta) \mathrm{d} \theta\right)=F\left(\tilde{\theta}^{F}(b)\right)$.

Now, $D^{G}(b) \geqslant D^{H}(b)$ iff $\int_{\theta_{A}}^{\tilde{\theta}^{H}(b)} H(\theta) \mathrm{d} \theta \geqslant \int_{\theta_{A}}^{\tilde{\theta}^{G}(b)} G(\theta) \mathrm{d} \theta$. If $G$ FSD $H$, then $\int_{\theta_{A}}^{\tilde{\theta}(b)} H(\theta) \mathrm{d} \theta \geqslant \int_{\theta_{A}}^{\tilde{\theta}(b)} G(\theta) \mathrm{d} \theta$. Thus, were $\tilde{\theta}^{G}(b)=\tilde{\theta}^{H}(b)$, then $D^{G}(b) \geqslant D^{H}(b)$ for high bids too. Nonetheless, it has been previously shown that under $\operatorname{SSD} \tilde{\theta}^{G}(b)>\tilde{\theta}^{H}(b)$, so that the ranking between $D^{G}(b)$ and $D^{H}(b)$ is ambiguous. One could in principle ex- 
pect that the dominance effect dominates the $\tilde{\theta}$ effect, and hence to get $D^{G}(b) \geqslant D^{H}(b)$. Nonetheless, we cannot conclude that this is always the case.

Expected revenues can be derived by employing the distribution function of the secondorder statistic, $D^{F(2)}(b)$, with $R^{F}=\int_{0}^{\bar{B}} b \mathrm{~d} D^{F(2)}(b)$. Note that if the distribution of equilibrium bids under $H$ dominates the one under $G$, i.e., $D^{H}(b) \leqslant D^{G}(b)$, then $D^{H(2)}(b) \leqslant$ $D^{G(2)}(b)$ does also hold, and hence expected revenues will be larger under $H$ than under $G$, i.e., $R^{H} \geqslant R^{G}$, even though the distribution $H$ is stochastically dominated by $G$. Thus, if $D^{H}(b) \leqslant D^{G}(b)$, then seller's expected revenues are larger when bidders are less likely to face severe financial constraints, even though the opposite holds when considering equilibrium bids.

In what follows we show that this is the case for a large family of distributions. We now focus on the following family of log-concave distributions, $F(\theta ; a)=(\theta-1)^{a}$ with support [1,2]. Note that $F\left(\theta ; a_{1}\right)$ strictly FSD $F\left(\theta ; a_{2}\right)$ iff $a_{1}>a_{2}$. Let $G(\theta)=F\left(\theta ; a_{1}\right)$ and $H(\theta)=F\left(\theta ; a_{2}\right)$ with $a_{1}>a_{2}$, so that $G$ FSD $H$. Within this family, we can explicitly derive $\tilde{\theta}^{H}(b)$. In particular, for any bid in $\left[\widehat{B}^{F}, \bar{B}\right]$,

$$
\tilde{\theta}^{H}(b)=\frac{a_{1}-a_{2}+\tilde{\theta}^{G}(b)\left(a_{2}+1\right)\left(a_{1}+2\right)}{\left(a_{2}+2\right)\left(a_{1}+1\right)},
$$

which is easily obtained from

$$
\tilde{\theta}^{H}(b)+\frac{\int_{1}^{\tilde{\theta}^{H}(b)} H(z) \mathrm{d} z}{H\left(\tilde{\theta}^{H}(b)\right)}=\tilde{\theta}^{G}(b)+\frac{\int_{1}^{\tilde{\theta}^{G}(b)} G(z) \mathrm{d} z}{G\left(\tilde{\theta}^{G}(b)\right)} .
$$

For high bids, we showed that $D^{G}(b) \geqslant D^{H}(b)$ iff $\int_{\theta_{A}}^{\tilde{\theta}^{H}(b)} H(\theta) \mathrm{d} \theta \geqslant \int_{\theta_{A}}^{\tilde{\theta}^{G}(b)} G(\theta) \mathrm{d} \theta$. After substituting for the specific $G$ and $H$ and integrating, the inequality above simplifies to

$$
\left(a_{1}+1\right)\left(\tilde{\theta}^{H}(b)-1\right)^{a_{2}+1} \geqslant\left(a_{2}+1\right)\left(\tilde{\theta}^{G}(b)-1\right)^{a_{1}+1},
$$

or, after substituting $\theta^{H}(b)$ with its value as derived in Eq. (5),

$$
\left(a_{1}+1\right)\left(\frac{\left(a_{2}+1\right)\left(a_{1}+2\right)}{\left(a_{2}+2\right)\left(a_{1}+1\right)}\right)^{a_{2}+1}\left(\theta^{G}(b)-1\right)^{a_{2}+1} \geqslant\left(a_{2}+1\right)\left(\theta^{G}(b)-1\right)^{a_{1}+1} .
$$

As $G$ FSD $H$, we know that $\left(\tilde{\theta}^{G}(b)-1\right)^{a_{2}+1} \geqslant\left(\tilde{\theta}^{G}(b)-1\right)^{a_{1}+1}$. Consequently, a sufficient condition to ensure that $D^{G}(b) \geqslant D^{H}(b)$ holds is given by

$$
\left(a_{1}+1\right)\left(\frac{\left(a_{2}+1\right)\left(a_{1}+2\right)}{\left(a_{2}+2\right)\left(a_{1}+1\right)}\right)^{a_{2}+1} \geqslant\left(a_{2}+1\right) .
$$

Straightforward operations allow us to rewrite the inequality above as

$$
\left(\frac{a_{1}+2}{a_{2}+2}\right)^{a_{2}+1} \geqslant\left(\frac{a_{2}+1}{a_{1}+1}\right)^{a_{2}} \text {. }
$$

Since $\left(a_{1}+2\right) /\left(a_{2}+2\right) \geqslant 1 \geqslant\left(a_{2}+1\right) /\left(a_{1}+1\right)$ whenever $a_{1}>a_{2}$, Eq. (6) holds as claimed. ${ }^{32}$

\footnotetext{
32 In Appendix B, we also provide an example with a uniform and a quadratic distribution, in which expected revenues are explicitly computed and compared. There too we derive the same result.
} 


\section{Conclusions}

The literature has demonstrated that second-price, common-value auctions are sensitive to the presence of asymmetries among bidders. For example, Bikhchandani (1988) has shown that if it is common knowledge that a bidder has a disadvantage compared to her opponent, that bidder (almost surely) never wins the auction. We show that this result does not carry over when one allows for two-sided uncertainty. Even if the probabilities that one of the bidders is an advantaged type while the other is a disadvantaged type are arbitrarily large, in every equilibrium, the disadvantaged type bidder needs to win the auction with strictly positive probability.

We then solve for the equilibria in two cases, one with two types and another with a continuum of types. We show that in the two-type case there is no symmetric equilibrium in continuous strategies and we construct an equilibrium in asymmetric and discontinuous strategies, while in the continuum-of-types case we construct a symmetric equilibrium in continuous strategies. We derive the convergence properties of both equilibria, and we show that they converge to the symmetric equilibria of the corresponding symmetric auctions. This is also true when considering convergence in terms of expected revenue performance. In other words, we reestablish a lost linkage in the analysis of pure-commonvalue and almost-common-value auctions. We thus highlight the fact that the presumed sensitivity to the presence of asymmetries was delivered due to specific characteristics in the extensions considered in the literature.

Finally, we are able to address the related but distinct literature of auctions under financially constrained bidders. We show in the continuum-of-types model that when bidders operate in a more financially constrained environment they bid more aggressively because their winner's curse gets attenuated. I.e., we are able to generalize the result derived in Fang and Parreiras (2002), in a model where the financial constraints were taking the specific form of budget constraints.

\section{Acknowledgments}

Comments and suggestions by Mark Armstrong, Luis Corchón, Paul Klemperer, JeanJacques Laffont, Jean Tirole, Mark Walker, the editor in charge and an anonymous referee, as well as participants at the 1st Game Theory World Congress in Bilbao, at ESEM and EEA 2001 in Lausanne, at ASSET 2001 in Crete, and at seminars in Madrid, Athens and Barcelona are gratefully acknowledged. The research started when the second author was at IDEI, Toulouse. All remaining errors are ours.

\section{Appendix A}

Throughout the paper, the analysis was based on the assumption that the efficiency handicap among different types of bidders, i.e., the range of $\theta$, is not large enough to outweigh the importance of the signals the bidders receive about the value of the object (i.e., the $x$ 's). For example, the proposition in the continuum-of-types case relies on the 
fact that $\left(0, \theta_{A}\right)$-bidder always bids zero, as her efficiency advantage does not outweigh her informational disadvantage. If $\theta_{D}>2$ though, then $\left(0, \theta_{A}\right)$-bidder must win over any $\left(x, \theta_{D}\right)$-type. In this case, the equilibrium needs to be modified. ${ }^{33}$

Consider the following example: let $x$ be uniformly distributed on $[0,1]$, and $\theta$ be uniformly distributed on $[1,4]$. We claim that the following strategies constitute a symmetric equilibrium:

$$
b^{*}(x, \theta)= \begin{cases}\frac{3 x}{3 \theta-4} & \text { if } x \in\left[0, \frac{3 \theta-4}{8}\right], 4 / 3<\theta \leqslant 4, \\ \frac{2 x+1}{2 \theta} & \text { if } x \in\left[\frac{3 \theta-4}{8}, \frac{\theta-1}{2}\right], 1<\theta<4 \\ \frac{3 x+1}{3 \theta-1} & \text { if } x \in\left[\frac{\theta-1}{2}, 1\right], 1<\theta<3\end{cases}
$$

Further, type $(0,4 / 3)$ randomizes over the bids in the interval $[0,3 / 8]$.

Proof. Assume that bidder I follows this strategy, and consider the best reply of bidder II. No matter what her $\theta$ is, she maximizes her expected profit by bidding up to the point in which she is indifferent about whether she is selected as a winner, when she is involved in a tie. Now, the true value of the object, when bidding $b$ and facing a tie, is $\left(x_{I I}+x_{I}^{E}(b)\right) / \theta_{I I}$, where $x_{I}^{E}(b)$ stands for the expected value of her opponent's signal in the event of a tie when bidding $b$. Since her opponent iso-bids are linear and distributions are uniform, then

$$
x_{I}^{E}(b)= \begin{cases}\frac{M(b)}{2} & \text { if } b \in\left[0, \frac{3}{8}\right], M(b) \text { such that } b^{*}(M(b), 4)=b, \\ \frac{1}{2} & \text { if } b \in\left[\frac{3}{8}, \frac{1}{2}\right], \\ \frac{m(b)+1}{2} & \text { if } b \in\left[\frac{1}{2}, 2\right], m(b) \text { such that } b^{*}(m(b), 1)=b .\end{cases}
$$

As $b^{*}(x, \theta)$ is equal to this expression, $\left(b^{*}, b^{*}\right)$ constitutes a symmetric equilibrium in continuous strategies.

Figure A.1 provides a graphical illustration of the equilibrium bids for $\bar{\theta}=1, \bar{\theta}=5 / 4$, $\bar{\theta}=3 / 2, \bar{\theta}=2, \bar{\theta}=5 / 2, \bar{\theta}=3$, and $\bar{\theta}=4$.

\section{Appendix B}

This appendix derives explicitly seller's expected revenues and compares them in an example with a quadratic and a uniform distribution for the $\theta$ s. Let $G(\theta)=\left(\theta^{2}-1\right) / 3$, and $H(\theta)=\theta-1$, where $\theta \in[1,2]$. Note that $G$ FSD $H$, as intended.

It is easy to see that the c.d.f. of equilibrium bids under $H$ is given by

$$
D^{H}(b)= \begin{cases}\frac{3 b}{4} & \text { if } 0 \leqslant b \leqslant \frac{4}{5}, \\ \frac{1}{9} b^{-1}(8-b)(2 b-1) & \text { if } \frac{4}{5} \leqslant b \leqslant 2 .\end{cases}
$$

Expected revenues can be readily computed: $R^{H}=\int_{0}^{2} 2 b\left(1-D^{H}(b)\right) \mathrm{d} D^{H}(b)=0.4424$.

33 We would like to thank an anonymous referee for turning our attention to this case. 


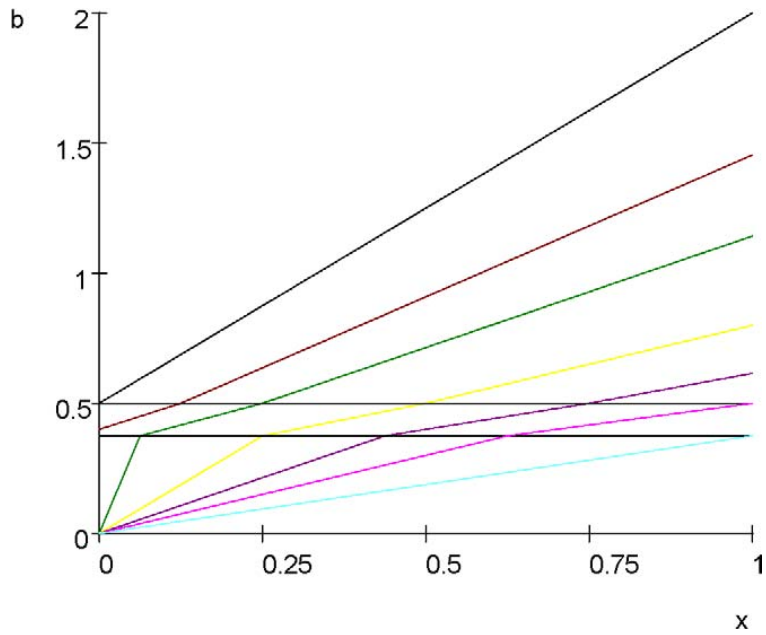

Fig. A.1. Bidding strategies.

Similarly, we get the c.d.f. of the equilibrium bids under $G$,

$$
D^{G}(b)= \begin{cases}\frac{7 b}{9} & \text { if } 0 \leqslant b \leqslant \frac{9}{11}, \\ \frac{b}{2}\left(\frac{1}{36 b^{3}} A^{3}-\frac{2}{9}\right)-\frac{1}{12 b^{2}} A^{2}+\frac{4}{3} & \text { if } \frac{9}{11} \leqslant b \leqslant 2,\end{cases}
$$

where $A=\left(\sqrt{3} \sqrt{4 b+4 b^{2}+3}-2 b+3\right) / 2$. In this case, expected revenues are $R^{G}=$ $\int_{0}^{2} 2 b\left(1-D^{G}(b)\right) \mathrm{d} D^{G}(b)=0.42665$. Therefore, expected revenues are larger under the uniform than under the quadratic distribution, i.e., expected revenues are larger when bidders are less financially constrained.

\section{References}

Bagnoli, M., Bergstrom, T., (1989). Log-concave probability and its applications. Working paper 89-23. University of Michigan, Center for Research on Economic and Social Theory.

Baxandall, P., Liebeck, H., 1986. Vector Calculus. Oxford Applied Mathematics and Computing Science Series. Clarendon Press, Oxford.

Bikhchandani, S., 1988. Reputation in repeated second-price auctions. J. Econ. Theory 46, 97-119.

Börgers, T., Dustmann, C., 2003. Awarding telecom licenses: The recent European experiences. Econ. Pol. 18, $215-268$.

Bracewell, R., 1999. The Fourier Transform and Its Applications, third ed. McGraw-Hill, New York, NY.

Bulow, J., Klemperer, P., 2002. Prices and the winner's curse. RAND J. Econ. 33, 1-21.

Bulow, J., Huang, M., Klemperer, P., 1999. Toeholds and takeovers. J. Polit. Economy 107, 427-454.

Che, Y.-K., Gale, I., 1998. Standard auctions with financially constrained bidders. Rev. Econ. Stud. 65, 1-25.

Dasgupta, P., Maskin, E., 2000. Efficient auctions. Quart. J. Econ. 115, 341-380.

Fang, H., Parreiras, S., 2002. Equilibrium of affiliated value second-price auctions with financially constrained bidders: The two-bidder case. Games Econ. Behav. 39, 215-236.

Jackson, M., (1999). The non-existence of equilibrium in auctions with two-dimensional types. Mimeo. Caltech. 
Klemperer, P., 1998. Auctions with almost common values: The "wallet game" and its applications. Europ. Econ. Rev. 42, 757-769.

Klemperer, P., 2002. What really matters in auction design. J. Econ. Perspect. 16, 169-189.

Maskin, E., Riley, J., 2000. Asymmetric auctions. Rev. Econ. Stud. 67, 413-438.

Milgrom, P., 1981. Rational expectations, information acquisition, and competitive bidding. Econometrica 49, 921-943.

Milgrom, P., Weber, R., 1982. A theory of auctions and competitive bidding. Econometrica 50, 1089-1122.

Ogryczak, W., Ruszczynski, A., 2002. Dual stochastic dominance and related mean-risk models. SIAM J. Optim. 13 (1), 60-78.

Royden, H.L., 1988. Real Analysis, third ed. MacMillan Co., New York, NY.

Zheng, C., 2001. High bids and broke winners. J. Econ. Theory 100, 129-171. 